\title{
Direction-Preserving Trajectory Simplification
}

\author{
Cheng Long ${ }^{\dagger}$, Raymond Chi-Wing Wong ${ }^{\dagger}$ \\ ${ }^{\dagger}$ The Hong Kong University of Science and Technology \\ \{clong, raywong\}@cse.ust.hk
}

\author{
H. V. Jagadish \\ ‡University of Michigan \\ jag@umich.edu
}

\begin{abstract}
Trajectories of moving objects are collected in many applications. Raw trajectory data is typically very large, and has to be simplified before use. In this paper, we introduce the notion of directionpreserving trajectory simplification, and show both analytically and empirically that it can support a broader range of applications than traditional position-preserving trajectory simplification. We present a polynomial-time algorithm for optimal directionpreserving simplification, and another approximate algorithm with a quality guarantee. Extensive experimental evaluation with real trajectory data shows the benefit of the new techniques.
\end{abstract}

\section{INTRODUCTION}

With the proliferation of GPS-embedded devices (e.g., smart phones and taxis), trajectory data is becoming ubiquitous. Indeed, it has been studied extensively in the past decades in the literature of Moving Objects Databases (MOD) [22, 19].Trajectory data is usually generated by periodically collecting the position of a moving object with the help of the GPS technologies.

Since the raw trajectory data is usually very large, simplifying trajectory data is important. To appreciate this, consider a city with $10 \mathrm{k}$ taxis. Suppose that we track the trajectory of each taxi by sampling its position once every 5 seconds (i.e., the sampling rate is $5 \mathrm{~s}$ ). The size of the collected trajectories for just one day is approximately 4 GB.

Raw trajectory data is large, and hence expensive to store. Even worse, it is expensive to manipulate and to analyze on account of its large size. In fact, most existing query processing and data mining algorithms on trajectory data are memory-resident and thus cannot be used with raw trajectory data that is too large to fit in memory.

A question one may ask is why not just sampling less frequently to reduce the size of the data. The answer is that, in real life, objects have great variance in their velocities. A taxi moving at 40 mph would have moved about 100 yards in $5 \mathrm{~s}$, whereas another taxi stuck at a traffic signal may not have moved at all. Obviously, we need more frequent observations of the former than of the latter. Similarly, we need more observations to capture a taxi that makes a turn and fewer for one that continues straight. Therefore, standard

Permission to make digital or hard copies of all or part of this work for personal or classroom use is granted without fee provided that copies are not made or distributed for profit or commercial advantage and that copies bear this notice and the full citation on the first page. To copy otherwise, to republish, to post on servers or to redistribute to lists, requires prior specific permission and/or a fee. Articles from this volume were invited to present their results at The 39th International Conference on Very Large Data Bases, August 26th - 30th 2013, Riva del Garda, Trento, Italy.

Proceedings of the VLDB Endowment, Vol. 6, No. 10

Copyright 2013 VLDB Endowment 2150-8097/13/10... \$10.00. practice is to oversample initially, and then to simplify by eliminating observations that add little information.

In view of this, several algorithms have been developed for simplifying trajectory data $[17,6,21,18,11]$. All these algorithms make the natural assumption that the goal should be to simplify trajectories such that the position information captured in the simplified trajectories is "similar" to the position information captured in the original trajectories. We can call them position-preserving trajectory simplification algorithms. However, as we will soon see, this objective, though natural, is not the best choice in many situations. To illustrate, let us work through a toy example in detail.

ExAmple 1 (Motivating ExAmple). Consider three raw trajectories $T_{1}, T_{2}$ and $T_{3}$ as shown in bold lines in Figure 1(a)(i), (a)(ii) and (a)(iii), respectively. Each of these trajectories has four positions, $p_{1}, p_{2}, p_{3}$ and $p_{4}$. $T_{1}$ and $T_{2}$ are similar to each other, and each of them is dissimilar to $T_{3}$. Thus, a trajectory clustering algorithm, such as [15], should group $T_{1}$ and $T_{2}$ in the same cluster and place $T_{3}$ by itself in a separate cluster.

Now suppose that these raw trajectories are too large, and so must be simplified to three points each before being further processed. We could use an existing position-preserving trajectory simplification, denoted by $\mathcal{A}_{\text {pos }}$, for this simplification. Following existing studies, the first position $p_{1}$ and the last position $p_{4}$ in each trajectory have to be kept. Therefore, one of position $p_{2}$ and position $p_{3}$ is to be retained, and the other one dropped.

Consider the simplification process on $T_{1}$. It can drop either $p_{2}$ or $p_{3}$ in the simplified trajectory. Let $d_{1}\left(d_{2}\right)$ be $p_{2}$ 's $\left(p_{3}\right)$ 's perpendicular distance to line segment $\overline{p_{1} p_{3}}\left(\overline{p_{2} p_{4}}\right)$. Since $d_{1}>d_{2}$, $\mathcal{A}_{\text {pos }}$ drops $p_{3}$ and returns the simplified trajectory $T_{1}^{\prime}$ as shown in Figure 1(b)(i). Similarly, $\mathcal{A}_{\text {pos }}$ return $T_{2}^{\prime}$ (Figure 1(b)(ii)) and $T_{3}^{\prime}$ (Figure 1(b)(iii)) as the simplified trajectories of $T_{2}$ and $T_{3}$, respectively. We now see that, though raw trajectories $T_{1}$ and $T_{2}$ are similar, their simplified trajectories $T_{1}^{\prime}$ and $T_{2}^{\prime}$ generated by $\mathcal{A}_{\text {pos }}$ are dissimilar. On the other hand, raw trajectories $T_{1}$ and $T_{3}$ are dissimilar, but their simplified trajectories $T_{1}^{\prime}$ and $T_{3}^{\prime}$ generated by $\mathcal{A}_{\text {pos }}$ are similar. In consequence, the clustering algorithm on the simplified trajectories $T_{1}^{\prime}, T_{2}^{\prime}$ and $T_{3}^{\prime}$, places $T_{1}$ and $T_{3}$ together into one cluster, and thus fails to produce correct (or expected) clusters.

In contrast, as will be shown later, a direction-preserving trajectory simplification method we introduce below, denoted by $\mathcal{A}_{d i r}$, would simplify $T_{1}, T_{2}$ and $T_{3}$ to $T_{1}^{\prime \prime}$ (Figure 1(c)(i)), $T_{2}^{\prime \prime}$ (Figure 1(c)(ii)) and $T_{3}^{\prime \prime}$ (Figure 1(c)(iii)), respectively. Since $T_{1}^{\prime \prime}$ and $T_{2}^{\prime \prime}$ are similar to each other and each of them is dissimilar to $T_{3}^{\prime \prime}$, the clustering algorithm based on these simplified trajectories would produce the expected clusters.

Before we can discuss direction-preserving trajectory simplification in depth, we first have to describe what direction information is, which we do next. 

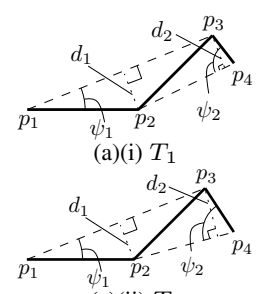

(a)(ii) $T_{2}$

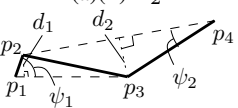

(a)(iii) $T_{3}$

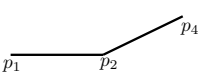

(b)(i) $T_{1}^{\prime}$

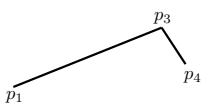

(b)(ii) $T_{2}^{\prime}$

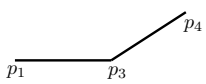

(b)(iii) $T_{3}^{\prime}$

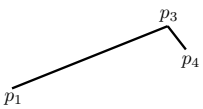

(c)(i) $T_{1}^{\prime \prime}$

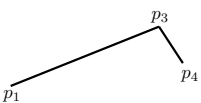

(c)(ii) $T_{2}^{\prime \prime}$

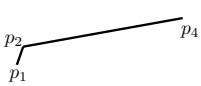

(c)(iii) $T_{3}^{\prime \prime}$

Figure 1: A motivating example

\subsection{Direction Information}

When an object moves from position $p$ to position $p^{\prime}$, we define the direction of this movement to be the angle of an anticlockwise rotation from the positive $\mathrm{x}$-axis to a vector from $p$ to $p^{\prime}$. The directions of all movements captured in the trajectory is called the direction information, and is used heavily, both directly and indirectly, in a wide range of applications on trajectory data. We list some of them as follows.

- Map Matching [1]. Given a digital map of a road network and a trajectory of an object moving on the road network, the map matching problem is to locate the trajectory on the digital map. Since each road segment in the road network has its own orientation, restricting the directions of the movements in the trajectory, the direction information plays an essential role in most map matching algorithms [1].

- Knowledge Discovery on Trajectory Data. As with other types of data, a rich set of knowledge discovery tasks has been proposed on the trajectory data [7]. Among them, many algorithms rely heavily on the direction information, which include [15, 9] for Clustering, [13] for Outlier Detection and [14] for Classification.

- Direction-based Query Processing. Sometimes, there are reasons to query trajectory information directly. One example is to find the trajectories moving within a direction range in a given time slot [2]. Another example is to find trajectories similar to a given trajectory, where the similarity measurement is based solely on moving direction [20].

In short, there are many situations in which direction preservation is important. Furthermore, as we show analytically in Section 3 and empirically in Section 6, direction preservation is stronger than position preservation, in that a simplification that preserves direction information well can be shown to preserve position information also, within some reasonable bounds. However, the converse is not true: position-preserving simplifications can be very bad at direction preservation.

\subsection{Direction-Preserving Trajectory Simplifi- cation (DPTS)}

In this paper, we propose a new trajectory simplification mechanism called Direction-Preserving Trajectory Simplification (DPTS) such that the direction information loss due to the simplification process is bounded. Within DPTS, we propose a direction-based measurement $E_{d}$, which is new and is defined to measure the error of a simplified trajectory in terms of the direction information. Let $T$ be a trajectory and $T^{\prime}$ be a simplification of $T$. The error (or simplification error) of $T^{\prime}$ under $E_{d}$, denoted by $\epsilon\left(T^{\prime}\right)$, is equal to the maximum angular difference between the direction of the movement during each time period in $T$ and the direction of the

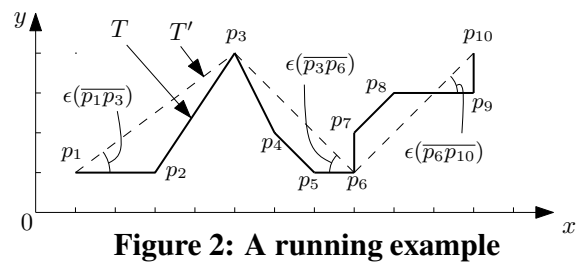

movement during the same time period in $T^{\prime}$. Then, the problem of DPTS is to simplify a given trajectory such that its size is minimized and its incurred simplification error (i.e., $\epsilon\left(T^{\prime}\right)$ ) is bounded by a given error tolerance $\epsilon_{t}$ where $\epsilon_{t} \in[0, \pi)$.

We use the maximum angular difference rather than the average angular difference to preserve better the shape of the trajectory. If we used the average one, we could still have a few segments that were completely off, resulting in the types of errors illustrated in Figure 1 for position-preserving techniques.

In this paper, we study the properties of DPTS, develop multiple algorithms to solve the DPTS problem, both exactly and approximately, and evaluate our algorithms experimentally. Specifically, we make the following contributions.

Contribution. First, we propose a novel notion of directionpreserving trajectory simplification, which favors a wide spectrum of applications on trajectory data. Second, we show that DPTS not only preserves direction information, but also preserves position information, thereby supporting a wide range of applications. Third, we adopt a common dynamic programming (DP) technique for DPTS. Since it is not scalable, we propose a novel optimal algorithm called $S P$ for DPTS. SP solves DPTS by first constructing a graph based on the given trajectory, then computing a shortest path in this graph and finally returning the solution for DPTS according to the shortest path found. The time complexity of $S P$ is $O\left(C \cdot n^{2}\right)$, where $C$ is usually a small constant $\left(C=1\right.$ if $\left.\epsilon_{t} \leq \pi / 2\right)$. Fourth, since even an $O\left(n^{2}\right)$ running time is likely to be unacceptable for a large $n$, we propose a scalable approximate algorithm called Intersect which runs in $O(n)$ time. We show that Intersect provides a certain degree of the quality guarantee in terms of the size of the simplified trajectory returned, in spite of running so fast. Finally, we perform a careful experimental comparison of these algorithms and a baseline using real trajectory data. The baseline is developed by common sense modifications of standard trajectory simplification techniques to address the DPTS problem.

The remainder of this paper is organized as follows. We define the DPTS problem in Section 2 and review the related work in Section 3. We introduce the optimal and approximate algorithms of DPTS in Section 4 and Section 5, respectively. We give the empirical study in Section 6 and conclude the paper in Section 7.

\section{PROBLEM DEFINITION}

A trajectory is represented by a sequence of $n$ triplets in the form of $\left(\left(x_{1}, y_{1}, t_{1}\right),\left(x_{2}, y_{2}, t_{2}\right), \ldots,\left(x_{n}, y_{n}, t_{n}\right)\right)$, where $\left(x_{i}, y_{i}\right)$ is the position in the 2D Euclidean space at time stamp $t_{i}$. We define positions $p_{i}=\left(x_{i}, y_{i}\right)$ for each $i \in[1, n]$. Then, $T$ 's trace is the sequence of ordered positions, i.e., $\left(p_{1}, p_{2}, \ldots, p_{n}\right)$.

Since the direction information of a trajectory is captured by its trace only, in the following, following existing studies, we focus on the trace part of the trajectory and use the terms "trajectory" and "trace" interchangeably. Thus, we simply denote $T$ by $\left(p_{1}, p_{2}, \ldots, p_{n}\right)$ by keeping the position information only. The size of $T$, denoted by $|T|$, is defined to be the number of positions in $T$.

Consider a running example as shown in Figure 2. In this figure, the trajectory $T$ is represented in the form of $\left(p_{1}, p_{2}, \ldots, p_{10}\right)$. The 


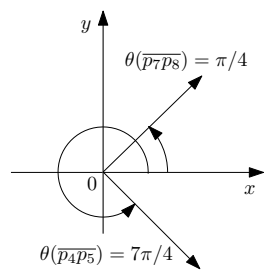

(a) (b)

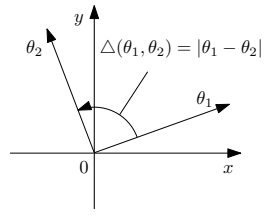

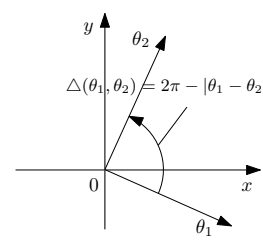

(c)
Figure 3: Examples illustrating the definition of "direction" and "angular difference"

size of this trajectory (i.e., $|T|$ ) is 10 . The start position of $T$ is $p_{1}$ and the end position of $T$ is $p_{10}$.

The straight line linking two positions $p_{i}$ and $p_{j}$ in $T$ where $1 \leq$ $i<j \leq n$ is denoted by $\overline{p_{i} p_{j}}$. If $p_{i}$ and $p_{j}$ are adjacent in $T$ (i.e., $j=i+1$ ), then $\overline{p_{i} p_{j}}$ is said to be a segment in $T$. Thus, a trajectory could also be regarded as a sequence of $n-1$ segments joining at $n-2$ positions (in addition to unique start and end positions).

In Figure 2, the solid horizontal straight line connecting $p_{1}$ and $p_{2}$ is denoted by $\overline{p_{1} p_{2}}$. Similarly, the dashed inclined straight line connecting $p_{1}$ and $p_{3}$ is denoted by $\overline{p_{1} p_{3}}$. Here, $\overline{p_{1} p_{2}}$ is a segment in $T$ but $\overline{p_{1} p_{3}}$ is not a segment in $T$. All segments in $T$ are shown in solid lines in the figure. In $T$, there are 9 segments jointing at 8 positions, namely $p_{2}, p_{3}, \ldots, p_{9}$.

Trajectory $T^{\prime}$ is said to be a simplification of $T$ if $T^{\prime}$ is of the form of $\left(p_{s_{1}}, p_{s_{2}}, \ldots, p_{s_{m}}\right)$ where $m \leq n$ and $1=s_{1}<s_{2}<$ $\ldots<s_{m}=n$. Note that $p_{1}$ and $p_{n}$ in $T$ must be kept in any simplification of $T$. There are $m-1$ segments in $T^{\prime}$, and $T^{\prime}$ is using $m-1$ segments to represent $T$ containing $n-1$ segments. For each $k \in[1, m)$, the segment $\overline{p_{s_{k}} p_{s_{k+1}}}$ in $T^{\prime}$ is used to approximate the sequence of segments between $p_{s_{k}}$ and $p_{s_{k+1}}$ in $T$, namely $\overline{p_{s_{k}} p_{s_{k}+1}}, \overline{p_{s_{k}+1} p_{s_{k}+2}}, \ldots, \overline{p_{s_{k+1}-1} p_{s_{k+1}}}$. In other words, this sequence of segments in $T$ is approximated by a single segment (i.e., $\overline{p_{s_{k}} p_{s_{k+1}}}$ ) in $T^{\prime}$ only.

Consider our running example. Let $T^{\prime}=\left(p_{1}, p_{3}, p_{6}, p_{10}\right) . T^{\prime}$ is a simplification of $T$ in Figure 2. Here, $s_{1}=1, s_{2}=3, s_{3}=6$ and $s_{4}=10$. Note that the size of $T^{\prime}$ is 4 . All segments in $T^{\prime}$ are shown in dashed lines in the figure. There are 3 segments in $T^{\prime}$. In other words, $T^{\prime}$ is using 3 segments to approximate 9 segments in $T$. Consider segment $\overline{p_{1} p_{3}}$ in $T^{\prime}$. It is used to approximate the sequence of segments between $p_{1}$ and $p_{3}$ in $T$, namely $\overline{p_{1} p_{2}}$ and $\overline{p_{2} p_{3}}$. In other words, $\overline{p_{1} p_{2}}$ and $\overline{p_{2} p_{3}}$ are approximated by a single segment $\overline{p_{1} p_{3}}$. Similarly, trajectory $T^{\prime \prime}=\left(p_{1}, p_{10}\right)$ is also a simplification of $T$, which uses only one segment (i.e., $\overline{p_{1} p_{10}}$ ) to approximate the whole trajectory $T$.

Direction-based Error Measurement $E_{d}$. Given a segment $\overline{p_{i} p_{i+1}}$ in $T$, the direction of $\overline{p_{i} p_{i+1}}$, denoted by $\theta\left(\overline{p_{i} p_{i+1}}\right)$, is defined to be the angle of an anticlockwise rotation from the positive $\mathrm{x}$-axis to a vector from $p_{i}$ to $p_{i+1}$. Thus, each direction falls in $[0,2 \pi)$. Consider our running example (Figure 2$). \theta\left(\overline{p_{7} p_{8}}\right)$ is $\pi / 4(=0.788)$ radian and $\theta\left(\overline{p_{4} p_{5}}\right)$ is $7 \pi / 4(=5.498)$ radian, as illustrated in Figure 3(a). It is easy to verify that $\theta\left(\overline{p_{1} p_{2}}\right)$ is equal to 0 radian, $\theta\left(\overline{p_{2} p_{3}}\right)$ is equal to 0.983 radian $\left(=\tan ^{-1} 3 / 2\right)$ and $\theta\left(\overline{p_{1} p_{3}}\right)$ is equal to 0.644 radian $\left(=\tan ^{-1} 3 / 4\right)$.

The angular difference between two directions $\theta_{1}$ and $\theta_{2}$, denoted by $\triangle\left(\theta_{1}, \theta_{2}\right)$, is defined to be the minimum of the angle of the anticlockwise rotation from $\theta_{1}$ to $\theta_{2}$ and that from $\theta_{2}$ to $\theta_{1}$, i.e.,

$$
\triangle\left(\theta_{1}, \theta_{2}\right)=\min \left\{\left|\theta_{1}-\theta_{2}\right|, 2 \pi-\left|\theta_{1}-\theta_{2}\right|\right\}
$$

For illustration, Figure 3(b) shows the case where $\triangle\left(\theta_{1}, \theta_{2}\right)=$ $\left|\theta_{1}-\theta_{2}\right|$ and Figure 3(c) shows the case where $\triangle\left(\theta_{1}, \theta_{2}\right)=$ $2 \pi-\left|\theta_{1}-\theta_{2}\right|$. Note that the angular difference between any two directions falls in $[0, \pi]$.
Consider our running example. The angular difference between $\theta\left(\overline{p_{1} p_{2}}\right)$ and $\theta\left(\overline{p_{1} p_{3}}\right)$ is $|0-0.644|=0.644$ and that between $\theta\left(\overline{p_{2} p_{3}}\right)$ and $\theta\left(\overline{p_{1} p_{3}}\right)$ is $|0.983-0.644|=0.339$.

Let $T^{\prime}=\left(p_{s_{1}}, p_{s_{2}}, \ldots, p_{s_{m}}\right)$ be a simplification of $T$ The simplification error of $T^{\prime}$ under $E_{d}$, denoted by $\epsilon\left(T^{\prime}\right)$, is defined as follows. Given a segment $\overline{p_{s_{k}} p_{s_{k+1}}}$ in $T^{\prime}$, the simplification error of $\overline{p_{s_{k}} p_{s_{k+1}}}$, denoted by $\epsilon\left(\overline{p_{s_{k}} p_{s_{k+1}}}\right)$, is defined to be the greatest angular difference between the direction of $\overline{p_{s_{k}} p_{s_{k+1}}}$ and the direction of a segment in $T$ approximated by $\overline{p_{s_{k}} p_{s_{k+1}}}$. That is,

$$
\epsilon\left(\overline{p_{s_{k}} p_{s_{k+1}}}\right)=\max _{s_{k} \leq h<s_{k+1}} \triangle\left(\theta\left(\overline{p_{s_{k}} p_{s_{k+1}}}\right), \theta\left(\overline{p_{h} p_{h+1}}\right)\right)
$$

Then, the simplification error of $T^{\prime}$ under $E_{d}$ is defined to be the greatest simplification error of a segment in $T^{\prime}$. That is,

$$
\epsilon\left(T^{\prime}\right)=\max _{1 \leq k<m} \epsilon\left(\overline{p_{s_{k}} p_{s_{k+1}}}\right)
$$

Consider back our running example (Figure 2). Each segment in $T^{\prime}$ has its simplification error. Consider the first segment $\overline{p_{1} p_{3}}$ in $T^{\prime}$ which approximates two segments in $T$, namely $\overline{p_{1} p_{2}}$ and $\overline{p_{2} p_{3}}$. Recall that $\triangle\left(\theta\left(\overline{p_{1} p_{3}}\right), \theta\left(\overline{p_{1} p_{2}}\right)\right)=0.644$ and $\triangle\left(\theta\left(\overline{p_{1} p_{3}}\right), \theta\left(\overline{p_{2} p_{3}}\right)\right)=0.339$. Thus, the simplification error of $\overline{p_{1} p_{3}}$ (i.e., $\epsilon\left(\overline{p_{1} p_{3}}\right)$ ) is equal to $\max \{0.644,0.339\}=0.644$. Similarly, we compute the simplification errors of the second segment $\overline{p_{3} p_{6}}$ and the third segment $\overline{p_{6} p_{10}}$ in $T^{\prime}$ which are both equal to 0.785 . Thus, the simplification error of $T^{\prime}$ in this example is equal to $\max \{0.644,0.785,0.785\}=0.785$.

In the following, when we write $\epsilon\left(\overline{p_{i} p_{j}}\right)(0 \leq i<j \leq n)$, we mean the simplification error of $\overline{p_{i} p_{j}}$ when it is used to approximate the line segments between $p_{i}$ and $p_{j}$ in $T$.

Problem Statement of DPTS. Let $T$ be a trajectory and $\epsilon_{t}$ be the error tolerance $\left(\epsilon_{t}<\pi\right)$. Trajectory $T^{\prime}$ is said to be an $\epsilon_{t^{-}}$ simplification of $T$ if $T^{\prime}$ is a simplification of $T$ and $\epsilon\left(T^{\prime}\right) \leq \epsilon_{t}$.

The DPTS problem is formalized as follows.

PROBLEM 1 (DPTS). Given a trajectory $T$ and an error tolerance $\epsilon_{t}$, the DPTS problem is to find the $\epsilon_{t}$-simplification of $T$ with the smallest size.

Consider our running example. Suppose that we set $\epsilon_{t}$ to 0.785 . $T^{\prime}$ is an $\epsilon_{t}$-simplification of $T$ since $\epsilon\left(T^{\prime}\right)=0.785 \leq \epsilon_{t}$. In fact, $T^{\prime}$ is the $\epsilon_{t}$-simplification of $T$ with the smallest size (which involves only four remaining positions).

\section{ANALYSIS OF PREVIOUS WORK}

We describe how DPTS relates to existing error measurements (Section 3.1) and trajectory simplification techniques (Section 3.2).

\subsection{Existing Error Measurements}

In this section, we show that the direction-preserving simplified trajectories give error guarantees in position-related properties, such as length and speed. However, the reverse is not true. That is, the position-preserving simplified trajectories $[17,18,21,11,6]$ do not give any error guarantee on the direction information.

Before we give our claims/properties (Section 3.1.2), we review some representative existing error measurements (Section 3.1.1).

\subsubsection{Existing Error Measurements}

Let $T=\left(p_{1}, p_{2}, \ldots, p_{n}\right)$ be a trajectory and $T^{\prime}=$ $\left(p_{s_{1}}, p_{s_{2}}, \ldots, p_{s_{m}}\right)$ be a simplification of $T(m \leq n)$. Several position-based measurements for evaluating the "simplification error" of $T^{\prime}$ have been defined in the literature. These measurements for $T^{\prime}$ are usually defined to be a distance measure which takes $T$ and $T^{\prime}$ as input. For each position $p_{h}$ of $T$ at the time stamp equal to $t_{h}$ where $1 \leq h \leq n$, the distance measure defines the estimated 
position of $p_{h}$, denoted by $p_{h}^{\prime}$, in $T^{\prime}$ based on some criteria. Let $d(\cdot, \cdot)$ be the Euclidean distance between two given points. Thus, the distance measure is defined to be $\max _{h \in[1, n]} d\left(p_{h}, p_{h}^{\prime}\right)$. Since different distance measures have different definitions on estimated positions, in the following, we focus on how to define estimated positions for some representative distance measures.

(1) Closest Euclidean Distance: With this distance measure, $p_{h}^{\prime}$ is defined to be the location on the segment $\overline{p_{s_{k}} p_{s_{k+1}}}$ of $T^{\prime}$ with $s_{k} \leq h \leq s_{k+1}$, which has the smallest Euclidean distance from $p_{h}$. We define a mapping function $M_{\mathcal{C}}$ which maps $p_{h}$ and $T^{\prime}$ to $p_{h}^{\prime}$ for this distance measure. That is, $p_{h}^{\prime}=M_{\mathcal{C}}\left(p_{h}, T^{\prime}\right)$.

(2) Synchronous Euclidean Distance: Under this distance measure, $p_{h}^{\prime}$ can be calculated with the following steps. The first step is to find the segment $\overline{p_{s_{k}} p_{s_{k+1}}}$ of $T^{\prime}$ with $s_{k} \leq h \leq s_{k+1}$. The second step is to find a point along the line passing through two points, namely $\left(x_{s_{k}}, y_{s_{k}}, t_{s_{k}}\right)$ and $\left(x_{s_{k+1}}, y_{s_{k+1}}, t_{s_{k+1}}\right)$, in a three-dimensional space such that the third dimensional value (representing the time dimension) of this point is $t_{h}$. Then, $p_{h}^{\prime}$ is set to be the first two-dimensional values of this point. Similarly, we define a mapping function $M_{\mathcal{S}}$ which maps both $p_{h}$ and $T^{\prime}$ to $p_{h}^{\prime}$. That is, $p_{h}^{\prime}=M_{\mathcal{S}}\left(p_{h}, T^{\prime}\right)$.

However, all of them adopt position-based distances instead of the direction-based distance studied in this paper. In the next section, we show that they do not give any error guarantee on the direction information.

\subsubsection{Theoretical Properties}

The length between two positions $p_{i}$ and $p_{j}$ wrt $T(i<j)$, denoted by $\operatorname{len}\left(p_{i}, p_{j} \mid T\right)$, is defined to be the length of the trace from $p_{i}$ to $p_{j}$ in $T$. That is,

$$
\operatorname{len}\left(p_{i}, p_{j} \mid T\right)=\sum_{k=i}^{j-1} d\left(p_{k}, p_{k+1}\right)
$$

The (average) speed between two positions $p_{i}$ and $p_{j} \operatorname{wrt} T(i<j)$, denoted by $\operatorname{speed}\left(p_{i}, p_{j} \mid T\right)$, is equal to $\operatorname{len}\left(p_{i}, p_{j} \mid T\right) /\left(t_{j}-t_{i}\right)$, where $t_{i}\left(t_{j}\right)$ is the time stamp corresponding to $p_{i}\left(p_{j}\right)$.

Interestingly, DPTS gives error guarantees on the length and speed information. Consider that $T$ is a trajectory and $T^{\prime}$ is an $\epsilon_{t}$-simplification of $T$ with $\epsilon_{t}<\pi / 2$. For any two adjacent positions $p_{i}$ and $p_{i+1}$ in $T$ where $i \in[1, n)$, both the length and the speed between the two corresponding estimated positions wrt $T^{\prime}$ are theoretically bounded. The estimated positions are determined by a mapping function. For the sake of space, in the remaining of the paper, if we do not specify the distance measure, we mean that we adopt the mapping function used in the Closest Euclidean Distance (i.e., $M_{\mathcal{C}}(\cdot, \cdot)$ ). The results based on the other mapping function (i.e., $M_{\mathcal{S}}(\cdot, \cdot)$ ) can be found in [16].

Lemma 1 (Bounded Length/SpeEd). Let $T$ be a trajectory and $T^{\prime}$ be an $\epsilon_{t}$-simplification of $T$ with $\epsilon_{t}<\pi / 2$. For any two adjacent positions $p_{i}$ and $p_{i+1}$ in $T$ where $i \in[1, n)$,

$$
\cos \left(\epsilon_{t}\right) \leq \frac{\operatorname{len}\left(p_{i}^{\prime}, p_{i+1}^{\prime} \mid T^{\prime}\right)}{\operatorname{len}\left(p_{i}, p_{i+1} \mid T\right)} \leq 1 \text { and } \cos \left(\epsilon_{t}\right) \leq \frac{\operatorname{speed}\left(p_{i}^{\prime}, p_{i+1}^{\prime} \mid T^{\prime}\right)}{\operatorname{speed}\left(p_{i}, p_{i+1} \mid T\right)} \leq 1
$$$$
\text { where } p_{i}^{\prime}=M_{\mathcal{C}}\left(p_{i}, T^{\prime}\right) \text { and } p_{i+1}^{\prime}=M_{\mathcal{C}}\left(p_{i+1}, T^{\prime}\right) \text {. }
$$

PROOF. Let $\overline{p_{s_{k}} p_{s_{k+1}}}$ be the segment of $T^{\prime}$ such that $p_{s_{k}}$ is the last position with $s_{k} \leq i$ and $p_{s_{k+1}}$ is the first position with $s_{k+1} \geq$ $i+1$. Consider Figure 4(a) for illustration. Since $\epsilon_{t}<\pi / 2$, we can verify that $p_{i}^{\prime}$ and $p_{i+1}^{\prime}$ are located along $\overline{p_{s_{k}} p_{s_{k+1}}}$.

Let $\psi_{i}$ be the angle formed by the two lines that pass through $\overline{p_{i} p_{i+1}}$ and $\overline{p_{s_{k}} p_{s_{k+1}}}$. Thus,

$$
\begin{aligned}
& \operatorname{len}\left(p_{i}^{\prime}, p_{i+1}^{\prime} \mid T^{\prime}\right)=d\left(p_{i}^{\prime}, p_{i+1}^{\prime}\right)=\cos \left(\psi_{i}\right) \cdot d\left(p_{i}, p_{i+1}\right) \\
& =\cos \left(\psi_{i}\right) \cdot \operatorname{len}\left(p_{i}, p_{i+1} \mid T\right) \geq \cos \left(\epsilon_{t}\right) \cdot \operatorname{len}\left(p_{i}, p_{i+1} \mid T\right)
\end{aligned}
$$
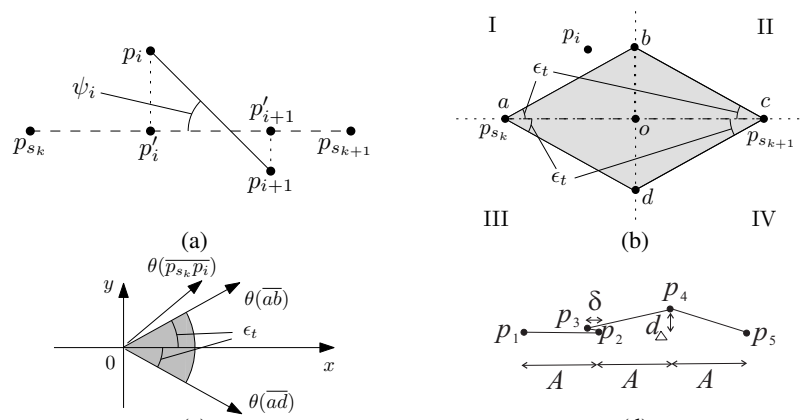

(c)

III

(b)

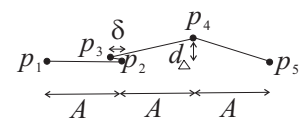

(d)

Figure 4: Proofs of Lemma 1, Lemma 2 and Lemma 3

which implies that $\cos \left(\epsilon_{t}\right) \leq \frac{\operatorname{len}\left(p_{i}^{\prime}, p_{i+1}^{\prime} \mid T^{\prime}\right)}{\operatorname{len}\left(p_{i}, p_{i+1} \mid T\right)} \leq 1$. Besides, since $\operatorname{speed}\left(p_{i}^{\prime}, p_{i+1}^{\prime} \mid T^{\prime}\right)=\operatorname{len}\left(p_{i}^{\prime}, p_{i+1}^{\prime} \mid T^{\prime}\right) /\left(t_{i+1}-t_{i}\right)$ and $\operatorname{speed}\left(p_{i}, p_{i+1} \mid T\right)=\operatorname{len}\left(p_{i}, p_{i+1} \mid T\right) /\left(t_{i+1}-t_{i}\right)$, we know $\cos \left(\epsilon_{t}\right) \leq \frac{\operatorname{speed}\left(p_{i}^{\prime}, p_{i+1}^{\prime} \mid T^{\prime}\right)}{\operatorname{speed}\left(p_{i}, p_{i+1} \mid T\right)} \leq 1$.

Interestingly, DPTS gives an error bound on the position information (in addition to the length/speed information).

LEMma 2 (BOUnded Position ERROR). Let $T$ be a trajectory and $T^{\prime}$ be an $\epsilon_{t}$-simplification of $T$ with $\epsilon_{t}<\pi / 2$. For each position $p_{i}$ in $T$ where $i \in[1, n]$, we have

$$
\begin{aligned}
& d\left(p_{i}, p_{i}^{\prime}\right) \leq 0.5 \cdot \tan \left(\epsilon_{t}\right) \cdot L_{\max } \\
& \text { where } p_{i}^{\prime}=M_{\mathcal{C}}\left(p_{i}, T^{\prime}\right) \text { and } L_{\max }= \\
& \max _{k \in[1, m)} \operatorname{len}\left(p_{s_{k}}, p_{s_{k+1}} \mid T^{\prime}\right) .
\end{aligned}
$$

PROOF. Let $\overline{p_{s_{k}} p_{s_{k+1}}}$ be the segment of $T^{\prime}$ such that $p_{s_{k}}$ is the last position with $p_{s_{k}} \leq i$ and $p_{s_{k+1}}$ is the first position with $p_{s_{k+1}} \geq i$. We construct a rhombus $\diamond_{a b c d}$ with four corners, namely $a, b, c$ and $d$, such that $a$ is at $p_{s_{k}}, c$ is at $p_{s_{k+1}}$ and the angle between $\overline{a b}(\overline{c b})$ and $\overline{a d}(\overline{c d})$ is equal to $2 \cdot \epsilon_{t}$. Consider Figure 4(b) for illustration where $\diamond_{a b c d}$ is indicated by the shaded area. We claim that $p_{i}$ is inside $\diamond_{a b c d}$ which we prove by contradiction.

Assume that $p_{i}$ is outside $\diamond_{a b c d}$. We partition the plane into 4 parts with the two lines that pass through $\overline{a c}$ and $\overline{b d}$ as indicated by I, II, III and IV in Figure 4(b), where $o$ is the intersection of the two lines. We consider 4 cases of which partition $p_{i}$ is in. Without loss of generality, suppose $p_{i}$ falls in part I.

Since $p_{i}$ is outside $\diamond_{a b c d}$, we know $\theta\left(\overline{p_{s_{k}}, p_{i}}\right)$ falls outside range $[\theta(\overline{a d}), \theta(\overline{a b})]$. For illustration, consider Figure 4(c).

Consider the segments between $p_{s_{k}}$ and $p_{i}$ in $T$. For each such segment $\overrightarrow{p_{h} p_{h+1}}\left(s_{k} \leq h<i\right)$, we denote by $\overrightarrow{p_{h} p_{h+1}}$ the vector from $p_{h}$ to $p_{h+1}$. We know that the direction of each such vector falls in range $[\theta(\overline{a d}), \theta(\overline{a b})]$ since otherwise $\epsilon\left(T^{\prime}\right)>\epsilon_{t}$. As a result, we know $\theta\left(\overline{p_{s_{k}} p_{i}}\right)$ falls in range $[\theta(\overline{a d}), \theta(\overline{a b})]$ since $\overrightarrow{p_{s_{k}} p_{i}}=\sum_{s_{k} \leq h<i} \overrightarrow{p_{h} p_{h+1}}$. This, however, contradicts the fact that $\theta\left(\overline{p_{s_{k}} p_{i}}\right)$ falls outside range $[\theta(\overline{a d}), \theta(\overline{a b})]$.

Thus, we know that $p_{i}$ falls in $\diamond_{a b c d}$. Therefore, we have

$$
\begin{gathered}
d\left(p_{i}, p_{i}^{\prime}\right) \leq d(b, o)=\tan \left(\epsilon_{t}\right) \cdot d(a, o)=0.5 \cdot \tan \left(\epsilon_{t}\right) \cdot d(a, c) \\
=0.5 \cdot \tan \left(\epsilon_{t}\right) \cdot \operatorname{len}\left(p_{s_{k}}, p_{s_{k+1}} \mid T^{\prime}\right) \leq 0.5 \cdot \tan \left(\epsilon_{t}\right) \cdot L_{\max }
\end{gathered}
$$

which finishes the proof.

Next, we show that existing position-preserving simplified trajectories do not have bounds on the direction information.

LEMMA 3 (UNBOUNDED DIRECTION ERROR). Let $T$ be a trajectory and $T^{\prime}$ be a (direction-based) $\epsilon_{t}$-simplification of $T$ with $\epsilon_{t}<\pi / 2$. Let $T_{\mathcal{C}}$ be a (position-based) simplified trajectory of 
$T$ such that $\left|T_{\mathcal{C}}\right|=\left|T^{\prime}\right|$ and the error of $T_{\mathcal{C}}$ under the Closest Euclidean Distance is minimized. There exists a dataset such that $\epsilon\left(T_{\mathcal{C}}\right) \approx \pi$ and $\epsilon\left(T^{\prime}\right) \approx 0$.

PROOF. We prove by constructing a problem instance as shown in Figure $4(\mathrm{~d}) . T=\left(p_{1}, p_{2}, p_{3}, p_{4}, p_{5}\right)$ is a trajectory, where $p_{1}$, $p_{2}, p_{3}$ and $p_{5}$ are located at a horizontal line and $p_{4}$ has its perpendicular distance from this line equal to a small real number $d_{\triangle}$. Besides, $d\left(p_{2}, p_{3}\right)=\delta$ where $\delta<<d_{\triangle}$.

Suppose that we can only keep 4 positions in the simplified trajectory. In other words, we have to remove 1 position from the 5 positions. If we consider preserving the direction information, $p_{4}$ will be removed and thus $T^{\prime}=\left(p_{1}, p_{2}, p_{3}, p_{5}\right)$. Thus, $\epsilon\left(T^{\prime}\right)=\epsilon\left(\overline{p_{3} p_{5}}\right) \approx 0$. If we consider preserving the position information, $p_{2}$ will be removed and thus $T_{\mathcal{C}}=\left(p_{1}, p_{3}, p_{4}, p_{5}\right)$. Hence, $\epsilon\left(T_{\mathcal{C}}\right)=\epsilon\left(\overline{p_{1} p_{3}}\right)=\triangle\left(\theta\left(\overline{p_{1} p_{3}}\right), \theta\left(\overline{p_{2} p_{3}}\right)\right) \approx \pi$.

\subsection{Existing Trajectory Simplification}

Many trajectory simplification techniques have been proposed. We categorize them by the main idea employed in the algorithm as follows. They are Split [17, 6], Merge [21, 18], Greedy [11, 17] and Dead-Reckoning [12].Split is an approach which finds a position in a given trajectory, according to the heuristic value of the position, to split the whole trajectory into two sub-trajectories and continues the process iteratively on each of the split sub-trajectories which cannot be approximated by a line segment connecting its start position and its end position. Merge is an approach which finds two adjacent segments in a given trajectory, according to the heuristic value computed from these two adjacent segments, discards the position $p$ bridging these two segments, and create a segment connecting the non-bridging end position of one segment and the non-bridging end position of the other segment. It continues the process iteratively until discarding any position $p$ violates the error tolerance. Greedy is an approach which finds a sequence of the greatest number of consecutive segments to be discarded and create a segment connecting the two end positions of this sequence iteratively until discarding any sequence of 2 consecutive segments violates the tolerance constraint. Dead-Reckoning is an online algorithm which reads each position sequentially and determines whether this position is discarded or not according to a heuristic criterion.

The aforementioned ideas of Split, Merger and Greedy can be adapted to our DPTS problem. The only change is to change the error measurement to our simplification error (Equation (2)). However, they have their drawbacks. First, they cannot return optimal solutions. Second, as shown in our experiments, they are not efficient compared with our proposed Intersect algorithm. Details of the adaptation can be found in our technical report [16].

Other related studies include [3] which studies the error bounds of several queries on the simplified trajectories with bounded simplification errors mainly measured by the position information, [5] which studies the trajectory simplification problem with the consideration of the shape and also the semantic meanings of the trajectory, [4] which introduces a multi-resolution polygonal curve approximation (also called line simplification) algorithm for trajectory simplification, and [10] which studies the trajectory simplification problem where the trajectories are constrained to a road network. None of these studies pay attention to the direction information for trajectory simplification.

\section{FINDING OPTIMAL SOLUTION}

A naive solution for the DPTS problem is to traverse each possible simplification of $T$ with its simplification error at most $\epsilon_{t}$ and then to pick the one with the smallest size. Since the number of all

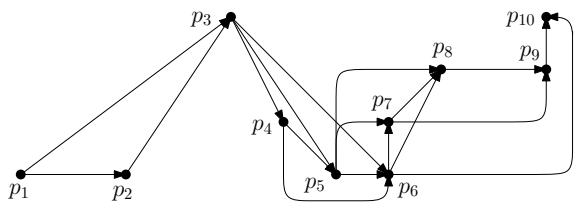

Figure 5: The graph $G_{\epsilon_{t}}$ constructed based on the running example when $\epsilon_{t}$ is set to be $\pi / 4=0.785$

possible simplifications of a trajectory $T$ is $2^{|T|-2}$, this solution is not feasible in practice. Alternatively, one may adopt a common dynamic programming (DP) technique for the DPTS problem. Unfortunately, the time complexity of this technique is cubic. For the sake of space, we include this DP algorithm in [16]. Instead, we propose a method called $S P$ which is much faster and scalable.

Algorithm SP involves the following three steps.

- Step 1 (Graph Construction): It first constructs a graph based on the given trajectory.

- Step 2 (Shortest Path Finding): It computes a shortest path in this graph.

- Step 3 (Solution Generation): It finally returns the solution for DPTS according to the shortest path found.

In Step 1, it constructs a graph wrt $\epsilon_{t}$, denoted by $G_{\epsilon_{t}}(V, E)$, as follows. For each position $p_{i}$ of $T$ where $1 \leq i \leq n$, it creates a vertex for $p_{i}$ in $V$. For each pair of two positions $\left(p_{i}, p_{j}\right)$ where $i<j$, it creates an edge $\left(p_{i}, p_{j}\right)$ in $E$ if $\epsilon\left(\overline{p_{i} p_{j}}\right) \leq \epsilon_{t}$.

In Step 2, it finds the shortest path from $p_{1}$ to $p_{n}$ in $G_{\epsilon_{t}}$ by a shortest path algorithm (e.g., a BFS search). Here, the length of a path is defined to be the number of edges involved along the path.

In Step 3, it generates the solution for DPTS according to the shortest path found. Note that all vertices involved in this shortest path correspond to all positions in the $\epsilon_{t}$-simplification of $T$ with the smallest size. Thus, if the ordering of the positions (or vertices) involved in the shortest path is " $p_{s_{1}}-p_{s_{2}}-\ldots-p_{s_{m}}$ ", it returns the solution $T^{\prime}$ as $\left(p_{s_{1}}, p_{s_{2}}, \ldots, p_{s_{m}}\right)$.

EXAmple 2 (Algorithm SP). Consider our running example in Figure 2. Suppose that $\epsilon_{t}=0.785$. In Step 1 of the $S P$ algorithm, we can construct graph $G_{\epsilon_{t}}$ accordingly as shown in Figure 5. In this figure, we construct a vertex for each position in $T$. Besides, for each pair of positions $p_{i}$ and $p_{j}$ where $i<j$, if $\epsilon\left(\overline{p_{i} p_{j}}\right) \leq \epsilon_{t}$, we create an edge $\left(p_{i}, p_{j}\right)$. Note that $\epsilon\left(\overline{p_{i} p_{i+1}}\right)=0$ for each $i \in[1, n-1]$.

In Step 2, we can find the shortest path in this graph. It is easy to verify that $p_{1}-p_{3}-p_{6}-p_{10}$ is the shortest path. Finally, in Step 3, we construct the solution of DPTS as $\left(p_{1}, p_{3}, p_{6}, p_{10}\right)$.

Let us analyze the time complexity of a straightforward implementation of algorithm SP. For Step 1, a straightforward solution for constructing $G_{\epsilon_{t}}$ is to try all possible pairs of $\left(p_{i}, p_{j}\right)$ where $1 \leq i<j \leq n$ and to check whether $\epsilon\left(\overline{p_{i} p_{j}}\right) \leq \epsilon_{t}$. Since there are $O\left(n^{2}\right)$ possible such pairs and the checking cost for each pair is $O(n)$, the time complexity of Step 1 is $O\left(n^{3}\right)$. For Step 2, a simple BFS could be adopted to find the shortest path from $p_{1}$ to $p_{n}$ in $G_{\epsilon_{t}}$, which takes $O(|V|+|E|)$ time. Since $|V|=O(n)$ and $|E|=O\left(n^{2}\right)$, we know that the cost of BFS is $O\left(n^{2}\right)$. Step 3 which returns the solution takes $O(n)$ time. As we can see, the time complexity of Step 1 (i.e., the graph construction) dominates those of Step 2 and Step 3. Thus, the overall time complexity of a straightforward implementation of algorithm $S P$ is $O\left(n^{3}\right)$. Besides, the space complexity of $S P$ is simply $O(|V|+|E|)$ which corresponds to the space cost of maintaining $G_{\epsilon_{t}}$.

In the following, we propose two kinds of enhancement techniques in order to improve the efficiency of our $S P$ algorithm. The 


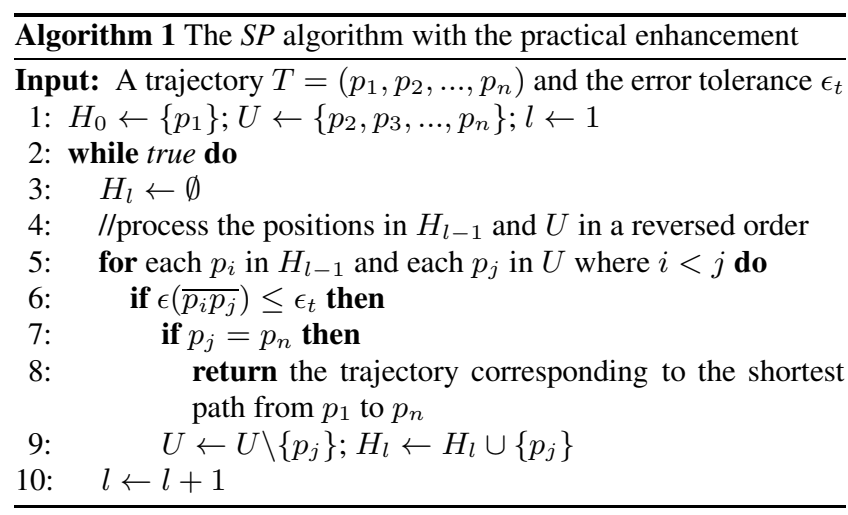

first one is called the practical enhancement (Section 4.1) which is to improve the performance of the algorithm in a practical way. The second one is called the complexity improvement (Section 4.2) which is to improve the theoretical time complexity of the algorithm from cubic to quadratic with some properties.

\subsection{Practical Enhancement}

The practical enhancement is to construct $G_{\epsilon_{t}}$ (in Step 1) on the demand of the BFS procedure used in Step 2. Specifically, the straightforward implementation has to materialize all possible edges in $G_{\epsilon_{t}}$ in Step 1 and then perform the BFS procedure in Step 2 . Here, the enhancement constructs only some of the edges in $G_{\epsilon_{t}}$ which are needed in the BFS procedure. Since some other edges need not be constructed, the space consumption can be reduced and some computations can be also saved.

Given a position $p$ in $T$ and a non-negative integer $l, p$ is said to be an l-length position if the length of the shortest path from $p_{1}$ to $p$ in $G_{\epsilon_{t}}$ is equal to $l$. Given a non-negative integer $l$, we define the l-length unique set, denoted by $H_{l}$, to be the set of all $l$-length positions in $T$. For example, the 0-length unique set $H_{0}$ is $\left\{p_{1}\right\}$. Consider the BFS procedure starting from $p_{1}$ on $G_{\epsilon_{t}}$. It first retrieves the set of positions which are the out-neighbors of $p_{1}$. This set corresponds to $H_{1}$. Then, starting from each position $p$ in $H_{1}$, it retrieves the set of positions which are the out-neighbors of $p$ and have not been retrieved before. This set corresponds to $\mathrm{H}_{2}$. The above process continues from $H_{2}$ in the same manner until $p_{n}$ is retrieved.

In view of the above discussion, we design our $S P$ algorithm with this enhancement as follows. We maintain the $l$-length unique sets $H_{l}(l=0,1,2, \ldots)$ which store the positions retrieved by the BFS procedure and $U$ for storing the remaining positions that have not been retrieved by the BFS procedure. We initialize $H_{0}$ to be $\left\{p_{1}\right\}$ and $U$ to be $\left\{p_{2}, p_{3}, \ldots, p_{n}\right\}$. We then compute $H_{l}$ based on $H_{l-1}$ for $l=1,2, \ldots$ iteratively as follows. We start from each position $p_{i}$ in $H_{l-1}$. For each position $p_{j}$ in $U$, we compute $\epsilon\left(\overline{p_{i} p_{j}}\right)$. If $\epsilon\left(\overline{p_{i} p_{j}}\right) \leq \epsilon_{t}$, we further check whether $p_{j}$ is $p_{n}$. If so, we stop the process since the shortest path from $p_{1}$ to $p_{n}$ has been found; otherwise, we exclude $p_{j}$ from $U$ and include it in $H_{l}$. Besides, when processing the positions in $H_{l-1}$ and $U$, we impose a reversed order, which corresponds to $p_{n}, p_{n-1}, \ldots, p_{1}$. The intuition is that we expect that $p_{n}$ could be retrieved earlier in this way. We present our enhanced $S P$ algorithm in Algorithm 1.

Complexity Analysis. The worst-case time complexity of the $S P$ algorithm with the practical enhancement keeps the same as that of the straightforward implementation, i.e., it is still $O\left(n^{3}\right)$. However, in practice, with the practical enhancement, the $S P$ algorithm is more efficient since some computations of $\epsilon\left(\overline{p_{i} p_{j}}\right)$ are avoided, and it is also more scalable since there is no need to materialize
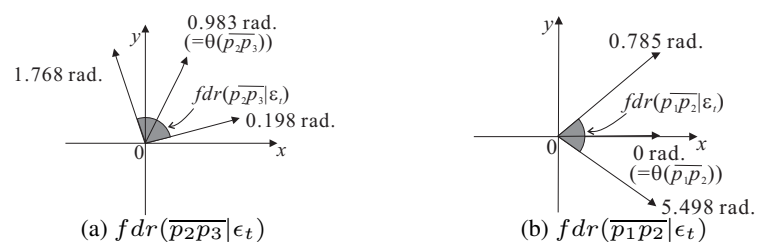

Figure 6: Illustration of $f d r\left(\overline{p_{2} p_{3}} \mid \epsilon_{t}\right)$ and $f d r\left(\overline{p_{1} p_{2}} \mid \epsilon_{t}\right)$

$G_{\epsilon_{t}}$. The space complexity of $S P$ with the practical enhancement is simply $O(n)$ since it maintains each position once and does not materialize $G_{\epsilon_{t}}$ explicitly.

\subsection{Complexity Improvement}

The complexity improvement is to improve the time complexity of our $S P$ algorithm from cubic to quadratic by using some properties for our algorithm. In this section, we focus on the complexity improvement based on the straightforward implementation for illustration. In Section 4.3, we describe how this complexity improvement can be incorporated with the practical enhancement.

As can be noticed, the cost of the straightforward implementation is dominated by the construction of graph $G_{\epsilon_{t}}$. In this section, we propose a technique to reduce the cost of constructing the graph from $O\left(n^{3}\right)$ to $O\left(C \cdot n^{2}\right)$ time, where $C$ is shown to be a small constant in most cases. The major idea of such an improvement is to reduce the time complexity of checking whether $\epsilon\left(\overline{p_{i} p_{j}}\right) \leq \epsilon_{t}$ (in the graph construction step) from $O(n)$ to $O(C)$ by utilizing a new concept called "feasible direction range". Before we present the main idea, we first introduce some related concepts.

Given two angles $\theta_{1}$ and $\theta_{2}$ in $[0,2 \pi)$, an angular range, represented in the form of $\left[\theta_{1}, \theta_{2}\right]$, is defined to be a set of all possible angles of a vector originated from the origin when it is rotated anti-clockwise from $\theta_{1}$ to $\theta_{2}$. For example, the shaded part in Figure 6(a) shows the angular range of $[0.198,1.768]$, and the shaded part in Figure 6(b) shows the angular range of [5.498, 0.785]. Since the direction of $\overline{p_{2} p_{3}}$ is 0.983 radian, we say that its direction is in $[0.198,1.768]$ but not in $[5.498,0.785]$. Similarly, since the direction of $\overline{p_{1} p_{2}}$ is 0 radian, we say that its direction is in [5.498, 0.785] but not in [0.198, 1.768].

DEFINITION 1 (FEASIBLE DIRECTION RANGE). Given $a$ segment $\overline{p_{h} p_{h+1}}(1 \leq h<n)$ in $T$, the feasible direction range of $\overline{p_{h} p_{h+1}} w r t \epsilon_{t}$, denoted by $f d r\left(\overline{p_{h} p_{h+1}} \mid \epsilon_{t}\right)$, is defined to be the angular range in the form of $\left[\theta_{1}, \theta_{2}\right]$ with $\theta_{1}=\left[\left(\theta\left(\overline{p_{h} p_{h+1}}\right)-\epsilon_{t}\right)\right.$ $\bmod 2 \pi]$ and $\theta_{2}=\left[\left(\theta\left(\overline{p_{h} p_{h+1}}\right)+\epsilon_{t}\right) \bmod 2 \pi\right]$.

The feasible direction range of $\overline{p_{h} p_{h+1}}$ wrt $\epsilon_{t}$ corresponds to a set of all possible directions each of which has its angular difference from $\overline{p_{h} p_{h+1}}$ at most $\epsilon_{t}$. We can write $f d r$ as follows.

$f d r\left(\overline{p_{h} p_{h+1}} \mid \epsilon_{t}\right)=\left[\theta\left(\overline{p_{h} p_{h+1}}\right)-\epsilon_{t}, \theta\left(\overline{p_{h} p_{h+1}}\right)+\epsilon_{t}\right] \bmod 2 \pi$

Consider our running example. Suppose that $\epsilon_{t}$ is set to 0.785 . Since the direction of $\overline{p_{2} p_{3}}$ is 0.983 radian, $f d r\left(\overline{p_{2} p_{3}} \mid \epsilon_{t}\right)=$ $([0.983-0.785,0.983+0.785] \bmod 2 \pi)=[0.198,1.768]$ (See Figure 6(a)). Similarly, since the direction of $\overline{p_{1} p_{2}}$ is 0 radian, $f d r\left(\overline{p_{1} p_{2}} \mid \epsilon_{t}\right)=([0-0.785,0+0.785] \bmod 2 \pi)=$ [5.498, 0.785] (See Figure 6(b)).

We denote by $T[i, j]$ the sub-trajectory of $T$ that is between position $p_{i}$ and position $p_{j}(1 \leq i<j \leq n)$, i.e., $T[i, j]=$ $\left(p_{i}, p_{i+1}, \ldots, p_{j}\right)$. We define the feasible direction range of a subtrajectory $T[i, j]$ wrt $\epsilon_{t}$, denoted by $f d r\left(T[i, j] \mid \epsilon_{t}\right)$, to be the intersection of the $f d r$ 's of the segments in $T[i, j]$. That is,

$$
f d r\left(T[i, j] \mid \epsilon_{t}\right)=\cap_{i \leq h<j} f d r\left(\overline{p_{h} p_{h+1}} \mid \epsilon_{t}\right)
$$




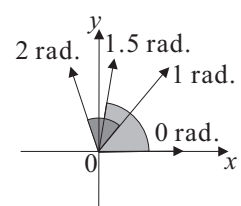

(a) $[0,1.5] \cap[1.0,2.0]$

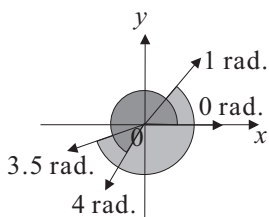

(b) $[0,4.0] \cap[3.5,1.0]$
Figure 7: Illustration of intersection operations between two angular ranges

In our running example, $f d r\left(T[1,3] \mid \epsilon_{r}\right)$ is equal to $\cap_{1 \leq h<3} f d r\left(\overline{p_{h} p_{h+1}} \mid \epsilon_{t}\right)=f d r\left(\overline{p_{1} p_{2}} \mid \epsilon_{t}\right) \cap f d r\left(\overline{p_{2} p_{3}} \mid \epsilon_{t}\right)=$ $[5.498,0.785] \cap[0.198,1.768]=[0.198,0.785]$. Figures 6(a) and (b) illustrate this scenario.

In the following, we simply write $f d r\left(\overline{p_{h} p_{h+1}}\right)(f d r(T[i, j]))$ for $f d r\left(\overline{p_{h} p_{h+1}} \mid \epsilon_{t}\right)\left(f d r\left(T[i, j] \mid \epsilon_{t}\right)\right)$ if the context of $\epsilon_{t}$ is clear.

In some cases, an intersection between an angular range and another angular range results in a single angular range. In some other cases, this intersection operation results in multiple disjoint angular ranges. To illustrate, an intersection between $[0,1.5]$ and $[1.0,2.0]$ results in a single angular range $[1,1.5]$ (as shown in Figure $7(a)$ ). An intersection between $[0,4.0]$ and $[3.5,1.0]$ results in two angular ranges $[3.5,4.0]$ and $[0,1.0]$ (as shown in Figure 7(b)).

Thus, from Equation (4), since $f d r(T[i, j])$ involves multiple intersection operations of angular ranges, it may consist of multiple disjoint angular ranges. We denote by $\|f d r(T[i, j])\|$ the number of disjoint angular ranges in $f d r(T[i, j])$.

With the concept of "feasible direction range", we are now ready to describe how we can check whether $\epsilon\left(\overline{p_{i} p_{j}}\right) \leq \epsilon_{t}$ efficiently.

LEMMA 4. Let $T=\left(p_{1}, p_{2}, \ldots, p_{n}\right)$ be a trajectory. $\epsilon\left(\overline{p_{i} p_{j}}\right) \leq$ $\epsilon_{t}$ iff $\theta\left(\overline{p_{i} p_{j}}\right)$ is in $f d r(T[i, j])$.

PROOF. "if": Assume that $\theta\left(\overline{p_{i} p_{j}}\right)$ is in $f d r(T[i, j])$. It follows that $\theta\left(\overline{p_{i} p_{j}}\right)$ is in $f d r\left(\overline{p_{h} p_{h+1}}\right)$ and thus $\triangle\left(\theta\left(\overline{p_{i} p_{j}}\right), \theta\left(\overline{p_{h} p_{h+1}}\right)\right) \leq \epsilon_{t}$ for $i \leq h<j$. Therefore, $\epsilon\left(\overline{p_{i} p_{j}}\right)=\max \left\{\triangle\left(\theta\left(\overline{p_{h} p_{h+1}}\right), \theta\left(\overline{p_{i} p_{j}}\right)\right) \mid i \leq h<j\right\} \leq \epsilon_{t}$.

"only-if": this direction could be verified similarly.

Lemma 4 suggests that checking whether $\epsilon\left(\overline{p_{i} p_{j}}\right) \leq \epsilon_{t}$ is equivalent to checking whether $\theta\left(\overline{p_{i} p_{j}}\right)$ is in $f d r(T[i, j])$. Suppose that $f d r(T[i, j])$ has been computed. Then, checking whether $\epsilon\left(\overline{p_{i} p_{j}}\right) \leq \epsilon_{t}$ takes $O(\|f d r(T[i, j])\|)$ only (compared with $O(n)$ in the straightforward implementation).

Note that in some cases, $\epsilon\left(\overline{p_{i} p_{j}}\right)>\epsilon_{t}$ but $\epsilon\left(\overline{p_{i} p_{j+k}}\right) \leq \epsilon_{t}$ where $j>i>0$ and $k>0$. By Lemma 4 , we know that $\theta\left(\overline{p_{i} p_{j}}\right)$ is not in $f d r(T[i, j])$ but $\theta\left(\overline{p_{i} p_{j+k}}\right)$ is in $f d r(T[i, j+k])$. To illustrate, in our running example (Figure 2), if we set $\epsilon_{t}=\pi / 4$, then $\epsilon\left(\overline{p_{6} p_{9}}\right)>\epsilon_{t}$ but $\epsilon\left(\overline{p_{6} p_{10}}\right) \leq \epsilon_{t}$. By Lemma $4, \theta\left(\overline{p_{6} p_{9}}\right)$ is not in $f d r(T[6,9])$ but $\theta\left(\overline{p_{6} p_{10}}\right)$ is in $f d r(T[6,10])$.

Now, we know that the checking step can be done in $O(\|f d r(T[i, j])\|)$. There are two remaining issues related to this checking step. The first issue is related to the size of $f d r(T[i, j])$. If this size is very large, the checking step is still expensive. Fortunately, we find that this size is usually a small constant. When $\epsilon_{t} \leq \pi / 2$, it is equal to 1 . The second issue is how to compute $f d r(T[i, j])$ efficiently for each $i$ and $j$ where $i<j$.

\subsubsection{Issue 1: Size of $f d r(T[i, j])$}

LEMMA 5. Let $T=\left(p_{1}, p_{2}, \ldots, p_{n}\right)$ be a trajectory and $\epsilon_{t}$ be the error tolerance. Then, given two integers $i$ and $j(1 \leq i<j \leq$ $n)$, $\|f d r(T[i, j])\|$ is bounded by $\min \left\{1+\left\lfloor\frac{\epsilon_{t}}{\left(\pi-\epsilon_{t}\right)}\right\rfloor, j-i\right\}$.

PROOF. We first give some concepts based on an angular range and provide a lemma which is used to prove Lemma 5.
We denote the universe angular range $[0,2 \pi)$ by $\mathcal{U}$, Given an angular range $[a, b]$, we denote its complement wrt $\mathcal{U}$, which is the angular range $(b, a)$, by $[a, b]^{c}$. We define the span of an angular range $[a, b]$, denoted by $[a, b] . s p a n$, to be equal to $(b-a)($ $\bmod 2 \pi)$. We define the span of $f d r(T[i, j])(1 \leq i<j \leq n)$, denoted by $f d r(T[i, j])$.span, to be equal to the sum of the spans of the disjoint angular ranges that are involved in $f d r(T[i, j])$.

LEMMA 6. Let $[a, b]$ and $\left[a^{\prime}, b^{\prime}\right]$ be two angular ranges. $[a, b] \cap$ $\left[a^{\prime}, b^{\prime}\right]$ involves two disjoint angular ranges iff $\left[a^{\prime}, b^{\prime}\right]^{c}$ (i.e., $\left.\left(b^{\prime}, a^{\prime}\right)\right)$ falls in $[a, b]$ completely.

PROOF. This could be verified easily by the fact that $[a, b] \cap$ $\left[a^{\prime}, b^{\prime}\right]$ involves two disjoint angular ranges, namely $\left[a, b^{\prime}\right]$ and $\left[a^{\prime}, b\right]$, iff $\left(b^{\prime}, a^{\prime}\right)$ falls in $[a, b]$ completely.

Suppose $f d r\left(\overline{p_{k} p_{k+1}}\right)$ is $\left[a_{k}, b_{k}\right]$ for $i \leq k<j$. Then, $f d r(T[i, j])$ is equal to $\cap_{k=i}^{j-1}\left[a_{k}, b_{k}\right]$. Besides, we know $\left[a_{k}, b_{k}\right]$. span $=2 \epsilon_{t}$ for $i \leq k<j$ since $a_{k}=\theta\left(\overline{p_{k} p_{k+1}}\right)-\epsilon_{t}$ $\bmod 2 \pi$ and $b_{k}=\theta\left(\overline{p_{k} p_{k+1}}\right)+\epsilon_{t} \bmod 2 \pi($ Definition 1$)$.

First, we prove $\|f d r(T[i, j])\| \leq j-i$ by induction of $k=$ $j-i$. Base step: $k=1$. The correctness is obvious since $f d r(T[i, i+1])=\left[a_{i}, b_{i}\right]$ which involves one angular range only (i.e., $\left.\left[a_{i}, b_{i}\right]\right)$ and thus $\|f d r(T[i, i+1])\|=1$. Induction step: $\| f d r(T[i, i+k) \| \leq k$ implies $\| f d r(T[i, i+k+1) \| \leq k+1$. Assume $\| f d r(T[i, i+k) \|=r(r \leq k)$. Specifically, let $\left[a_{1}^{\prime}, b_{1}^{\prime}\right],\left[a_{2}^{\prime}, b_{2}^{\prime}\right], \ldots,\left[a_{r}^{\prime}, b_{r}^{\prime}\right]$ be the $r$ disjoint angular ranges involved in $f d r(T[i, i+k])$. Then, $f d r(T[i, i+k+1])$, which is equal to $f d r(T[i, i+k]) \cap\left[a_{i+k}, b_{i+k}\right]$, corresponds to $r$ intersections, $\left[a_{h}^{\prime}, b_{h}^{\prime}\right] \cap\left[a_{i+k}, b_{i+k}\right]$ for $1 \leq h \leq r$, each two of which are disjoint. Among these $r$ intersections, we show that at most one involves two disjoint angular ranges by contradiction. Assume that there exists $h_{1}$ and $h_{2}\left(i \leq h_{1} \neq h_{2} \leq r\right)$ such that both $\left[a_{h_{1}}^{\prime}, b_{h_{1}}^{\prime}\right] \cap\left[a_{i+k}, b_{i+k}\right]$ and $\left[a_{h_{2}}^{\prime}, b_{h_{2}}^{\prime}\right] \cap\left[a_{i+k}, b_{i+k}\right]$ involve two disjoint angular ranges. According to Lemma 6, we know that $\left(b_{i+k}, a_{i+k}\right)$ is in both $\left[a_{h_{1}}^{\prime}, b_{h_{1}}^{\prime}\right]$ and $\left[a_{h_{2}}^{\prime}, b_{h_{2}}^{\prime}\right]$, which leads to a contradiction since $\left[a_{h_{1}}^{\prime}, b_{h_{1}}^{\prime}\right]$ and $\left[a_{h_{2}}^{\prime}, b_{h_{2}}^{\prime}\right]$ are disjoint. As a result, $f d r\left(T\left[i, i+k_{1}\right]\right)$ involves at most $r+1$ disjoint angular ranges. That is, $\|f d r(T[i, i+k+1])\| \leq r+1 \leq k+1$.

Second, we prove $\|f d r(T[i, j])\| \leq 1+\left\lfloor\frac{\epsilon_{t}}{\pi-\epsilon_{t}}\right\rfloor$. We compute $f d r(T[i, i+1]), f d r(T[i, i+2]), \ldots, f d r(T[i, j])$ sequentially based on the following equation.

$$
\begin{aligned}
f d r(T[i, i+k+1]) & =f d r(T[i, i+k]) \cap\left[a_{i+k}, b_{i+k}\right] \\
& =f d r(T[i, i+k]) \backslash\left(b_{i+k}, a_{i+k}\right)
\end{aligned}
$$

We have two cases regarding Equation 5. Case 1: $\| f d r(T[i, i+$ $k+1])\|=\| f d r(T[i, i+k]) \|+1$. In this case, according to Lemma $6,\left(b_{i+k}, a_{i+k}\right)$ is in one of the disjoint angular ranges that are involved in $f d r(T[i, i+k+1])$. Then, we deduce that $f d r(T[i, i+k+1]) . s p a n=f d r(T[i, i+k]) . s p a n-$ $\left(2 \pi-2 \epsilon_{t}\right)$ since $\left(a_{i+k}-b_{i+k}\right) \bmod 2 \pi=2 \pi-2 \epsilon_{t}$. Case 2 : $\| f d r(T[i, i+k+1)\|=\| f d r(T[i, i+k) \|$. In this case, we have $f d r(T[i, i+k+1])$. span $\leq f d r(T[i, i+k])$.span since the span is non-increasing after an intersection operation. In view of the above two cases, we conclude that the increase of the number of disjoint angular ranges by 1 (in Case 1 only) is due to the decrease of the span by $\left(2 \pi-2 \epsilon_{t}\right)$. Since at the beginning, $\|f d r(T[i, i+1])\|=1$ and $f d r(T[i, i+1]) . \operatorname{span}=2 \epsilon_{t},\|f d r(T[i, i+k])\|$ has its greatest value equal to $\left(1+\left\lfloor\frac{2 \epsilon_{t}}{2 \pi-2 \epsilon_{t}}\right\rfloor\right)$.

According to Lemma $5,\|f d r(T[i, j])\|$ is usually bounded by a small constant. Let $C=\min \left\{1+\left\lfloor\frac{\epsilon_{t}}{\left(\pi-\epsilon_{t}\right)}\right\rfloor, j-i\right\}$. In particular, when $\epsilon_{t} \leq \pi / 2,\left\lfloor\frac{\epsilon_{t}}{\left(\pi-\epsilon_{t}\right)}\right\rfloor$ is equal to 0 . In this case, $\|f d r(T[i, j])\|$ is exactly equal to $\min \{1, j-i\}=1$ (since $j>i$ ). 


\subsubsection{Issue 2: How to Compute $f d r(T[i, j])$ Efficiently}

A straightforward method to compute $f d r(T[i, j])$ is to compute $f d r\left(\overline{p_{h} p_{h+1}}\right)(i \leq h<j)$ independently and then to intersect these $f d r$ 's. This straightforward method, nevertheless, incurs the cost of $\Omega(n)$ on average. Since we have $\Theta\left(n^{2}\right)$ instances of $f d r(T[i, j])$, computing all instances of $f d r(T[i, j])$ with this method incurs the total cost of $\Omega\left(n^{3}\right)$. Fortunately, this method could be improved significantly since it involves a lot of redundant work. A better method only takes $O(C)$ instead of $\Omega(n)$ to compute $f d r(T[i, j])$ based on the following incremental property.

Given two integers $i$ and $j$ where $1 \leq i<j<n$,

$$
f d r(T[i, j+1])=f d r(T[i, j]) \cap f d r\left(\overline{p_{j} p_{j+1}}\right)
$$

Suppose that the content of $f d r(T[i, j])$ is known, since the total number of angular ranges in $f d r\left(\overline{p_{j} p_{j+1}}\right)$ is 1 , we can compute $f d r(T[i, j+1])$ in $O(C)$ time. Note that $C$ is the greatest number of angular ranges in $f d r(T[i, j])$ and the intersection operation between two intervals could be finished in $O(1)$ time.

Thus, we propose to compute $f d r(T[i, j])(1 \leq i<j \leq n)$ using the incremental property. Specifically, it involves $n$ rounds.

- At round 1, it computes $f d r(T[h, h+1])$ (i.e., $f d r\left(\overline{p_{h} p_{h+1}}\right)$ ) for $1 \leq h<n$.

- At round $r(r>1)$, it computes $f d r(T[h, h+r])$ for $1 \leq h \leq n-r$. Specifically, it computes $f d r(T[h, h+r])$ by intersecting $f d r(T[h, h+r-1])$ (which has been maintained at round $r-1)$ and $f d r\left(\overline{p_{h+r-1} p_{h+r}}\right)$. Note that this operation takes $O(C)$ time.

Complexity Analysis. The time complexity of the above method for computing $f d r(T[i, j])$ 's for $1 \leq i<j \leq n$ is $O\left(C \cdot n^{2}\right)$ since it involves $n$ rounds and each round incurs the cost of $O(C \cdot n)$. Since there are $O\left(n^{2}\right)$ times of checking whether $\epsilon\left(\overline{p_{i} p_{i+1}}\right) \leq \epsilon_{t}$ and each checking can be done in $O(C)$ time with the $f d r(T[i, j])$ information, the time complexity of the $S P$ algorithm with the complexity improvement is $O\left(C \cdot n^{2}\right)$. Besides, the above method of computing $f d r(T[i, j])$ 's has its space complexity of $O(C \cdot n)$ since at each round, it is sufficient to maintain $O(n) f d f(T[i, j])$ 's each of which involves $O(C)$ intervals. Since the $S P$ algorithm with the complexity improvement materializes $G_{\epsilon_{t}}$ explicitly, we know that its space complexity is $O(C \cdot n+|V|+|E|)$, where $V$ $(E)$ is the vertex (edge) set of $G_{\epsilon_{t}}$.

\subsection{Combining The Two Enhancements}

The practical enhancement involves the construction of only some edges, which means that we just need to perform the checking of $\epsilon\left(\overline{p_{i} p_{j}}\right) \leq \epsilon_{t}$ for some pairs of positions $\left(p_{i}, p_{j}\right)$ only where $1 \leq i<j \leq n$. However, the cost of checking whether $\epsilon\left(\overline{p_{i} p_{j}}\right) \leq \epsilon_{t}$ is $O(n)$ which is expensive. In contrast, the complexity improvement reduces the cost of checking whether $\epsilon\left(\overline{p_{i} p_{j}}\right) \leq \epsilon_{t}$ from $O(n)$ to $O(C)$, but the undesired part is that it always computes $f d r(T[i, j])$ for all pairs of positions $\left(p_{i}, p_{j}\right)$.

In this part, we propose to unify the good aspects of both the practical enhancement and the complexity improvement and at the same time, to avoid their undesired aspects. Our strategy is to maintain $f d r(T[i, j])(1 \leq i<j \leq n)$ (i.e., the idea of complexity improvement) on the demand of the BFS process (i.e., the idea of the practical enhancement). Specifically, when checking whether $\epsilon\left(\overline{p_{i} p_{j}}\right) \leq \epsilon_{t}(1 \leq i<j \leq n)$, we have two cases. Case 1: $f d r(T[i, j])$ has been computed. In this case, the checking could be finished in $O(C)$ time. Case 2: $f d r(T[i, j])$ has not been computed. In this case, we recursively resort to $f d r(T[i, j-1])$ for computing $f d r(T[i, j])$. This recursive process stops either in Case 1 or $f d r(T[i, i+1])$ is acquired. Note that $f d r(T[i, i+1])$ could be computed in $O(1)$ time by Equation (3).
This version of $S P$ algorithm enjoys the benefit of the practical enhancement since it checks whether $\epsilon\left(\overline{p_{i} p_{j}}\right) \leq \epsilon_{t}(1 \leq i<$ $j \leq n$ ) on demand of the BFS process and does not materialize $G_{\epsilon_{t}}$ explicitly, and it also enjoys the benefit of the complexity improvement since it adopts the "feasible direction range" concept for checking whether $\epsilon\left(\overline{p_{i} p_{j}}\right) \leq \epsilon_{t}$, which is fast.

It could be verified that the worse-case time complexity of the $S P$ algorithm with both the practical enhancement and the complexity improvement is $O\left(C \cdot n^{2}\right)$ since it computes only a sub-set of all possible $f d r(T[i, j])$ 's.

Besides, the space complexity of this version of $S P$ is $O(C \cdot n)$ (it is sufficient to maintain for each $i$ the computed $f d r[i, j]$ with the largest $j$ among all computed $f d r[i, j]$ 's throughout the execution of the algorithm since each $f d r[i, j]$ is enquired at most once and note that it does not materialize $G_{\epsilon_{t}}$ ).

\section{FINDING APPROXIMATE SOLUTION}

According to the discussion in Section 4, the time complexity of an optimal algorithm for DPTS is at least quadratic. This, however, is not scalable enough when the datasets involves millions of positions. In this section, we develop an approximate algorithm called Intersect for the DPTS problem, which runs in linear time and gives a certain degree of quality guarantee.

Before we describe Intersect, we first give a concept of "feasibility" used in the algorithm. Given an error tolerance $\epsilon_{t}$ and two integers $i$ and $j$ where $1 \leq i<j \leq n, \overline{p_{i} p_{j}}$ is said to be $\epsilon_{t}$-feasible iff $f d r\left(T[i, j] \mid \epsilon_{t}\right)$ is non-empty. With this concept, we have the following property.

LEMMA 7 (FEASIBILITY). Given an error tolerance $\epsilon_{t}$ and two integers $i$ and $j$ where $1 \leq i<j \leq n$, if $\overline{p_{i} p_{j}}$ is $\frac{\epsilon_{t}}{2}$-feasible, then $\epsilon\left(\overline{p_{i} p_{j}}\right) \leq \epsilon_{t}$.

Proof SKetch. Since $\overline{p_{i} p_{j}}$ is $\frac{\epsilon_{t}}{2}$-feasible, $f d r\left(T[i, j] \mid \epsilon_{t} / 2\right)$ is non-empty. We deduce that for any two segments between $p_{i}$ and $p_{j}$ in $T, \overline{p_{h} p_{h+1}}$ and $\overline{p_{h^{\prime}} p_{h^{\prime}+1}}$ where $i \leq h<h^{\prime}<j$, $\triangle\left(\theta\left(\overline{p_{h} p_{h+1}}\right), \theta\left(\overline{p_{h^{\prime}} p_{h^{\prime}+1}}\right)\right) \leq \epsilon_{t}$. We further deduce that $\theta\left(\overline{p_{i} p_{j}}\right)$ is in $f d r\left(T[i, j] \mid \epsilon_{t}\right)$ because $\theta\left(\overline{p_{i} p_{j}}\right)$ is equal to the angle of a vector which is equal to the sum of all vectors between $p_{i}$ and $p_{j}$ in $T$ (i.e., $\sum_{k=i}^{j-1} \overrightarrow{p_{k} p_{k+1}}$ where $\overrightarrow{p_{k} p_{k+1}}$ is a vector from $p_{k}$ to $p_{k+1}$ for each $k \in[1, j-1]$ ) in the two-dimensional space (i.e., the $x$-coordinate and the $y$-coordinate). Here, the angle of a vector is defined to be the angle of an anticlockwise rotation from the positive $\mathrm{x}$-axis to this vector. By Lemma $4, \epsilon\left(\overline{p_{i} p_{j}}\right) \leq \epsilon_{t}$. The complete proof could be found in [16].

Specifically, Intersect has the following steps. Let $T^{\prime}$ be a variable storing the simplified trajectory to be returned. Let $e$ be a variable storing the position index of the last position in $T^{\prime}$. Let $h$ be a variable storing the position index of the position in $T$ being processed. Initially, Intersect initializes $T^{\prime}$ to be $\left(p_{1}\right)$, and then sets $e$ to be 1 ( ince $p_{1}$ is currently the last position in $T^{\prime}$ ). Then, it reads each of the remaining positions sequentially. It sets $h$ to 2 (since $p_{2}$ is the position in $T$ to process next). It proceeds with an iterative step as follows. Whenever $h \leq n$ and $\overline{p_{e} p_{h}}$ is $\frac{\epsilon_{t}}{2}$-feasible, it increments $h$ by 1 . It terminates this iterative step when either (1) $h>n$ or (2) $h$ has just been incremented to a value such that $\overline{p_{e} p_{h}}$ is not $\frac{\epsilon t}{2}$-feasible. For both stopping conditions, we know that $\overline{p_{e} p_{h-1}}$ is $\frac{\epsilon_{t}}{2}$-feasible and thus by Lemma 7, we have $\epsilon\left(\overline{p_{e} p_{h-1}}\right) \leq \epsilon_{t}$. Thus, $p_{h-1}$ is appended to $T^{\prime}$. Then, $e$ is set to $h-1$. It repeats the above iterative step whenever $h \leq n$. At the end, it returns $T^{\prime}$. The pseudo-code of Intersect is shown in Algorithm 2.

With Lemma 7, it is easy to verify that the trajectory returned by Intersect is an $\epsilon_{t}$-simplification of $T$. 


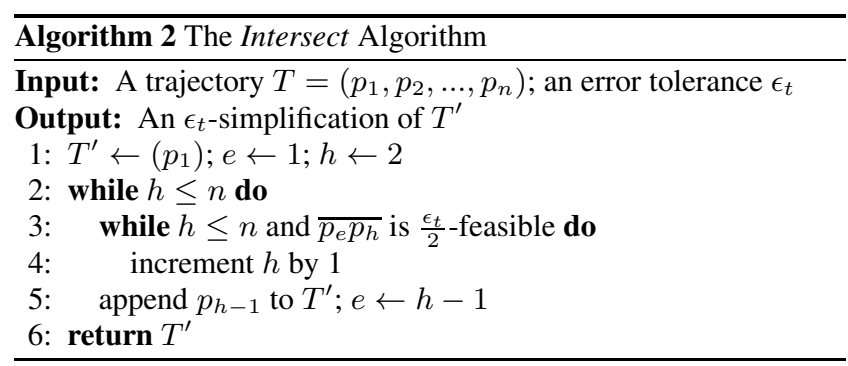

LEMMA 8. Let $T^{\prime}$ be the output of the Intersect algorithm in Algorithm 2. Then, $T^{\prime}$ is an $\epsilon_{t}$-simplification of $T$.

Intersect not only scans the data once only and returns an $\epsilon_{t^{-}}$ simplification of $T$ at the end, but also provides a certain degree of guarantee on the size of the simplified trajectory.

LEMMA 9 (SIZE BOUND). Let $T^{\prime}$ be the output of the Intersect algorithm in Algorithm 2. We have $\left|T^{\prime}\right| \leq\left|T^{\prime \prime}\right|$, where $T^{\prime \prime}$ is the $\epsilon_{t} / 2$-simplification of $T$ with the minimum size.

PROOF SKETCH. Let $T=\left(p_{1}, \ldots, p_{n}\right), T^{\prime}=\left(p_{s_{1}}, \ldots, p_{s_{m}}\right)$ and $T^{\prime \prime}=\left(p_{r_{1}}, \ldots, p_{r_{l}}\right)$. By definition, we have $s_{1}=r_{1}=1$ and $s_{m}=r_{l}=n$. Note that $n=|T|, m=\left|T^{\prime}\right|$ and $l=\left|T^{\prime \prime}\right|$. Our proof has two steps. First, we prove that $f d r\left(T\left[r_{k}, r_{k+1}\right] \mid \epsilon_{t} / 2\right)$ is non-empty for $1 \leq k<l$. This could be verified by the fact that $T^{\prime \prime}$ is an $\epsilon_{t} / 2$-simplification. Second, we prove $m \leq l$ by contradiction which main idea is that if $m>l$, we show $s_{k} \geq r_{k}$ for $k=1,2, \ldots, l$ by induction, which, however, results in that $s_{m}>n$ contradicting $s_{m}=n$. The complete proof could be found in [16].

Complexity Analysis. We know that variable $h$ is incremented whenever line 4 of Algorithm 2 is executed. Both the stopping condition of the outer while-loop (line 2) and one of the stopping conditions of the inner while-loop (line 3 ) are " $h \leq n$ ". We conclude that there are $O(n)$ times to execute the steps in line 3, line 4 and line 5. The step in line 4 and the step in line 5 take $O(1)$ time. In the following, we show that the step in line 3 also takes $\mathrm{O}(1)$ time. Thus, the time complexity of Intersect is $O(n)$.

The remaining issue is to derive the time complexity of the step in line 3. In line 3, checking whether $h \leq n$ can be done in $O(1)$ time. However, a straightforward implementation of checking whether $\overline{p_{e} p_{h}}$ is $\frac{\epsilon_{t}}{2}$-feasible (line 3) (or equivalently checking whether $f d r\left(T[e, h] \mid \epsilon_{t} / 2\right)$ is non-empty) is expensive. This is because as described in Section 4.2.2, computing $f d r\left(T[e, h] \mid \epsilon_{t} / 2\right)$ from scratch is expensive. By using the same technique in Section 4.2.2, we can perform this checking operation in $O(1)$ time. Specifically, we introduce a variable called fdr to store the content of $f d r\left(T[e, h] \mid \epsilon_{t} / 2\right)$. We have the following two changes in the algorithm due to this variable. Firstly, between line 2 and line 3 , we insert a statement that "fdr $\leftarrow f d r\left(\overline{p_{e} p_{h}} \mid \epsilon_{t} / 2\right)$ ". Note that $h=e+1$ at this moment, and the time complexity of this statement is $O\left(C^{\prime}\right)$ where $C^{\prime}=\min \left\{1+\left\lfloor\frac{\epsilon_{t} / 2}{\left(\pi-\epsilon_{t} / 2\right)}\right\rfloor, h-e\right\}$ (by using Lemma 5). Secondly, in the inner while-loop, just after line 4, we insert a statement that "fdr $\leftarrow \mathrm{fdr} \cap f d r\left(\overline{p_{h-1} p_{h}} \mid \epsilon_{t} / 2\right)$ ". Note that the time complexity of this statement is $O\left(C^{\prime}\right)$. With these two changes, checking whether $\overline{p_{e} p_{h}}$ is $\frac{\epsilon_{t}}{2}$-feasible (line 3 ) is equivalent to checking whether the current content of fdr is non-empty because the current content of fdr is equal to $f d r\left(T[e, h] \mid \epsilon_{t} / 2\right)$ (by Equation (6)). Furthermore, since $\epsilon_{t}$ is at most $\pi$ and thus $\epsilon_{t} / 2$ is at most $\pi / 2$, we deduce that $C^{\prime}=O(1)$. We conclude that the time complexity of the step in line 3 is $O(1)$.

\begin{tabular}{|c||c|c|c|c|}
\hline & $\begin{array}{l}\text { \# of tra- } \\
\text { jectories }\end{array}$ & $\begin{array}{l}\text { total \# of } \\
\text { positons }\end{array}$ & $\begin{array}{l}\text { average \# } \\
\text { of posi- } \\
\text { tions per } \\
\text { trajectory }\end{array}$ & $\begin{array}{l}\text { directional differ- } \\
\text { ence between two } \\
\text { adjacent segments } \\
\text { (Mean, S.D.) }\end{array}$ \\
\hline \hline Deer & 32 & 20,065 & 627 & $(1.669,0.948)$ \\
\hline Elk & 33 & 47,204 & 1,430 & $(1.647,0.984)$ \\
\hline Hurricane & 570 & 17,736 & 31 & $(0.213,0.300)$ \\
\hline Geolife & 17,621 & $24,876,978$ & 1,412 & $(0.364,0.615)$ \\
\hline T-Drive & 10,359 & $17,740,902$ & 1,713 & $(0.657,0.803)$ \\
\hline
\end{tabular}

Table 1: Real datasets

The space complexity of Intersect is $O(n)$ (which corresponds to the memory usage for storing the simplified trajectory $T^{\prime}$ ).

\section{EMPIRICAL STUDIES}

\subsection{Datasets and Algorithms}

We used 5 real datasets in our experiments, namely Deer, Elk, Hurricane, Geolife and T-Drive. Deer and Elk ${ }^{1}$ are two animal movement datasets which contain the radio-telemetry locations of deers in 1995 and elks in 1993, respectively. Hurricane ${ }^{2}$ contains the trajectories of the Atlantic hurricanes from year 1950 to year 2004. These three datases (i.e., Deer, Elk and Hurricane) are benchmark datasets for trajectory clustering [15]. Geolife ${ }^{3}$ records the outdoor movements of 182 users in a period of 5 years and TDrive $^{4}$ is a set of taxi trajectories in Beijing. These two datasets are widely-used for a broad range of applications on trajectory data [24, 23]. The statistics of these datasets are summarized in Table 1.

We study 5 optimal algorithms with the following notions. DP is the dynamic programming algorithm and SP is the straightforward implementation of the $S P$ algorithm. SP-prac (SP-theo) is the $S P$ algorithm with the practical enhancement (complexity improvement) only and SP-both is the one with all enhancements. Besides, we study 4 approximate algorithms, Split, Merge, Greedy and Intersect. The first three are the adaptations of the existing trajectory simplification methods and the forth is proposed in this paper.

All algorithms were implemented in $\mathrm{C} / \mathrm{C}++$ and run on a Linux platform with a $2.66 \mathrm{GHz}$ machine and 4GB RAM.

\subsection{Relevance to Existing Studies}

In this section, we conducted experiments to show how DPTS is relevant to existing studies.

\subsubsection{Bounds of DPTS wrt Existing Measurements}

In this part, we verify the theoretical bounds of the length (speed) error and the position error of DPTS as introduced in Section 3.1.2.

We vary the tolerance $\epsilon_{t}$ on $\{0.2,0.4,0.6,0.8,1\}$. The results about length (speed) errors are shown in Figure $8(\mathrm{a})$, where the "length (speed) ratio" is defined to be $\min _{i \in[1, n)}\left\{\operatorname{len}\left(p_{i}^{\prime}, p_{i+1}^{\prime} \mid T^{\prime}\right) / \operatorname{len}\left(p_{i}, p_{i+1} \mid T\right)\right\}$ where $p_{i}^{\prime}$ is the estimated position of $p_{i}$ on $T^{\prime}$ for $i \in[1, n]$. Thus, the larger this ratio is, the more accurate the length (speed) information of the simplified trajectory is. Note that the length ratio and the speed ratio (with respect to a segment in the original trajectory) are exactly the same since the speed is equal the length divided by the time difference between the time stamps of the two end-points of the segment, and the time difference in the original trajectory is kept to be the same as the time difference in the simplified trajectory. We observe that the theoretical bound of the length (speed) ratio is usually good (e.g., it is about 0.92 when $\epsilon_{t}=0.4$ ).

\footnotetext{
${ }^{1}$ http://www.fs.fed.us/pnw/starkey/data/tables/

${ }^{2}$ http://weather.unisys.com/hurricane/atlantic/

${ }^{3} \mathrm{http}: / /$ research.microsoft.com/en-us/downloads/b16d359d-d164469e-9fd4-daa38f2b2e13/

${ }^{4}$ http://research.microsoft.com/apps/pubs/?id=152883
} 
The results about the position error are shown in Figure 8(b). We observe that the empirical position error is usually significantly smaller than the theoretical bound (by near to one order of magnitude). Besides, when $\epsilon_{t}$ increases, the increase in the position error of DPTS becomes smaller. When $\epsilon_{t}$ becomes large, the position error of DPTS keeps quite stable.

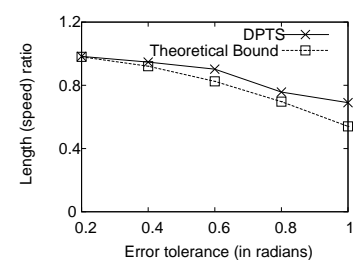

(a) Length and speed error

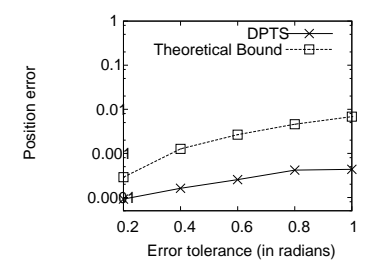

(b) position error
Figure 8: Verification of theoretical error bounds (Geolife)

\subsubsection{DPTS vs. PPTS}

In this part, we want to compare DPTS with $\underline{\text { Position-Preserving }}$ Trajectory Simplification (PPTS) in terms of two measurements, namely the position error and the direction error. In Section 3, we show that the position error of DPTS is bounded (Lemma 2) while the direction error of PPTS is un-bounded (Lemma 3). We study how worst the position error of DPTS compared with PPTS is and how worst the direction error of PPTS compared with DPTS is.

We adopt the Douglas-Peucker algorithm [6] for PPTS which is the most popular existing algorithm for PPTS [17, 3, 8], and we use our $S P$ algorithm for DPTS. We vary $\epsilon_{t}$ for DPTS. For a fair comparison, we enforce that the simplified trajectories from DPTS and PPTS have the same size. The results are shown in Figure 9(a) for position errors and in Figure 9(b) for direction errors. Consider Figure 9(a). It could be noticed that though the position errors of DPTS are usually larger than those of PPTS, the difference is small. For example, the ratio of the position errors is between 1.85 to 3 . Consider Figure 9(b). We observe that the direction errors of PPTS are significantly larger than that of DPTS. For example, when $\epsilon_{t}=$ 0.2 , the ratio is more than 10 . Besides, the direction errors of PPTS are greater than 2 , a value greater than $\pi / 2$, even with a small value of $\epsilon_{t}$ and is nearly to $\pi$, the greatest possible direction error, with a medium value of $\epsilon_{t}$, which implies that PPTS can hardly preserves the direction information. In conclusion, our DPTS preserves the direction information by its nature and also the position information to a certain degree, but PPTS preserves the position information only but not the direction information.
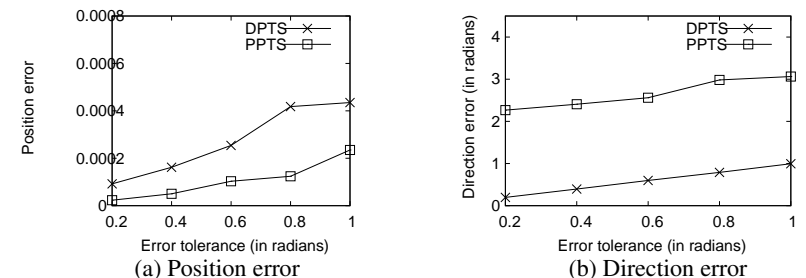

Figure 9: Comparison with existing PPTS (Geolife)

\subsubsection{An Application Study (Trajectory Clustering)}

In Section 6.2.2, we compared DPTS and PPTS with their favormetrics (i.e., the position error, favoring PPTS, and the direction error, favoring DPTS). In this section, we compare DPTS with PPTS with a neutral metric, the clustering quality, for a real-life application, trajectory clustering.

The main idea is as follows. Let $\mathcal{D}$ be a set of raw trajectories. We perform DPTS (PPTS) on each trajectory in $\mathcal{D}$ and obtain a set of simplified trajectories, denoted by $\mathcal{D}^{d}\left(\mathcal{D}^{p}\right)$. Then, we perform a clustering procedure on each of these two sets of trajectories and obtain the corresponding clustering results. We regard the clustering results based on $\mathcal{D}$ as ground truth and measure the qualities of the clustering results on $\mathcal{D}^{d}$ and $\mathcal{D}^{p}$. We verify DPTS by showing that the clustering results on $\mathcal{D}^{d}$ are consistently better (closer to the ground truth) than those on $\mathcal{D}^{p}$.

Consider the clustering procedure on $\mathcal{D}$ first. The clustering results based on $\mathcal{D}$ (i.e., the cluster membership of each trajectory) could be represented by a binary matrix $M_{n_{t} \times n_{c}}$, where $n_{t}$ is the number of trajectories in the set and $n_{c}$ is the number of resulting clusters. Note that under some clustering mechanisms such as the one in [15], a trajectory could belong to multiple clusters. For each trajectory $T \in \mathcal{D}$, its cluster membership could be represented by an $n_{c}$-dimensional binary vector. For each pair of trajectories $T_{1}$ and $T_{2}$ in $\mathcal{D}$, we measure the similarity between $T_{1}$ and $T_{2}$ by the Euclidean distance between $T_{1}$ 's cluster membership (which is a vector) and $T_{2}$ 's cluster membership (which is a vector). If the distance is below a pre-set threshold $\sigma$, we regard $T_{1}$ and $T_{2}$ to be similar; otherwise, we regard $T_{1}$ and $T_{2}$ to be dissimilar. Thus, based on the clustering results on $\mathcal{D}$, we can always obtain a similarity matrix which indicates for each pair of two trajectories in $\mathcal{D}$ whether they are similar or not. Let $S$ be such a similarity matrix corresponding to $\mathcal{D}$. The distance threshold $\sigma$ for deciding whether two trajectories are similar is set to 0.5 by default (all distances are normalized to $[0,1])$.

For a specific trajectory $T \in \mathcal{D}$, we denote its simplification in $\mathcal{D}^{d}$ and $\mathcal{D}^{p}$ by $T^{d}$ and $T^{p}$, respectively.

Similarly, we perform the same clustering procedure on $\mathcal{D}^{d}$ and $\mathcal{D}^{p}$ as we did on $\mathcal{D}$ and obtain their corresponding similarity matrices, denoted by $S^{d}$ and $S^{p}$, respectively.

We measure the quality of the clustering on $\mathcal{D}^{d}$ (and the clustering on $\mathcal{D}^{p}$ ) as follows. For a pair of two trajectories $T_{1}$ and $T_{2}$ in $\mathcal{D}$, we have 4 cases. Case 1: $T_{1}$ and $T_{2}$ are similar (wrt $S$ ) and $T_{1}^{d}$ and $T_{2}^{d}$ (the simplified trajectories of $T_{1}$ and $T_{2}$ under DPTS) are similar (wrt $S^{d}$ ). In this case, we have an occurrence of true positive (TP). Case 2: $T_{1}$ and $T_{2}$ are dissimilar (wrt $S$ ) and $T_{1}^{d}$ and $T_{2}^{d}$ are similar (wrt $S^{d}$ ). In this case, we have an occurrence of false positive (FP). Similarly, Case 3 and Case 4 correspond to the occurrences of false negative (FN) and true negative (TN), respectively. We adopt three measures in our experiments for measuring the clustering results. The first is called Rand, which is defined to be $(|T P|+|T N|) /(|T P|+|F P|+|F N|+|T N|)$ where $|\cdot|$ denotes the number of occurrences. The second and the third are defined to be $|T P| /(|T P|+|F P|)$ and $|T N| /(|T N|+|F N|)$, respectively. The larger the measure is, the better the clustering is.

For the trajectory clustering procedure, we adopt the TRACLUS algorithm [15] and the CATS algorithm [9]. According to [9], existing trajectory clustering algorithms fall in two categories. The first category includes those algorithms which take each trajectory as a whole for clustering while the second category includes the algorithms which use sub-trajectories for clustering. CATS is the state-of-the-art in the first category and TRACLUS is the state-ofthe-art in the second category [9].

For the PPTS procedure, again, we adopt the popular DouglasPeucker algorithm.

We vary the error tolerance $\epsilon_{t}$ with the values of $0.2,0.4,0.6,0.8$ and 1 for DPTS. Figure 10(a), (b) and (c) show the results about the Rand measure, the measure of $|T P| /(|T P|+|F P|)$ and the measure of $|T N| /(|T N|+|F N|)$ on the Deer dataset, respectively.

We observe that the clustering based on the simplified trajectories returned by DPTS is consistently better than that based on the simplified trajectories returned by PPTS. This might be explained by the fact that the direction information is heavily used in the trajectory clustering algorithms and the direction information loss due 


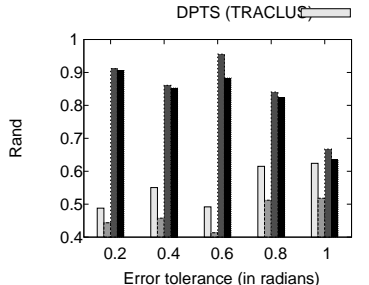

(a) Rand

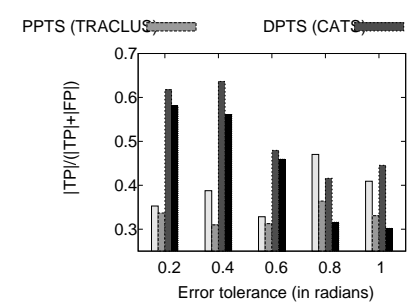

(b) $|T P| /(|T P|+|F P|)$

Figure 10: Trajectory Clustering Study (the Deer dataset)

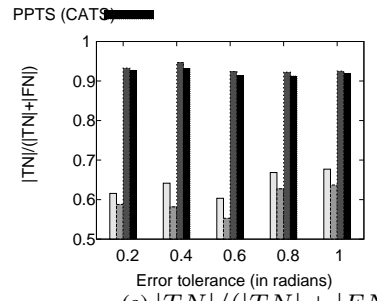

(c) $|T N| /(|T N|+|F N|)$

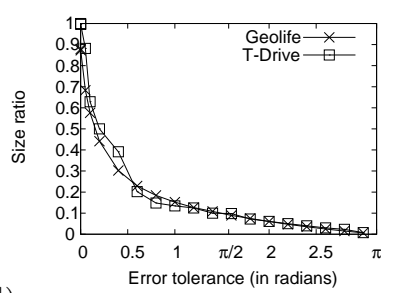

Figure 11: Compression rate to DPTS is bounded while that due to PPTS is un-bounded.

\subsection{Performance Study of the Optimal Algs}

In the part, we study the effects of 2 factors, namely the data size (i.e., $|T|$ ) and the error tolerance (i.e., $\epsilon_{t}$ ), on the performance of the optimal algorithms. We use 2 measures, namely the running time and the memory.

Effect of $|T|$. The values used for $|T|$ are around $2 k 4 k, 6 k, 8 k$ and $10 k$ ( $\epsilon_{t}$ is fixed to be 1 ). For each setting of $|T|$, we select a set of 10 trajectories each of which has its size near to this value and run DPTS on each of these trajectories. Then, we average the experimental results on these trajectories (this policy is used throughout our experiments without specification). Figure 12 show the results on Geolife. According to these results, SP-both is the fastest while $\mathrm{DP}$ is the slowest due to its high time complexity. Besides, the complexity improvement helps to reduce the running time dramatically (e.g., SP-theo is faster than SP by 2-3 orders of magnitude). This could be easily explained by the fact that with the complexity improvement, the cost of checking whether $\epsilon\left(\overline{p_{i} p_{j}}\right) \leq \epsilon_{t}$ is reduced from $O(n)$ to $O(C)$ ( $C$ is a small constant). Though the practical enhancement improves the time efficiency a little, it helps to reduce the memory significantly (e.g., the memory occupied by SP-theo is 1-3 orders of magnitude larger than that occupied by SP-both and the difference increases when $|T|$ increases on Geolife).

The experimental results on T-Drive are similar. Due to page limit, we put these results in the full version of this paper [16].

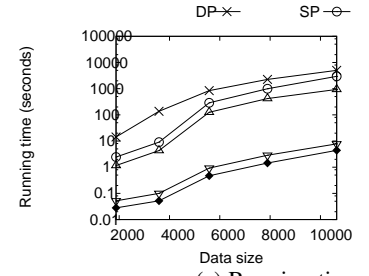

$$
\begin{aligned}
& \text { Data size } \\
& \text { (a) Running time }
\end{aligned}
$$

Figure 12. Fffec

$$
\text { }
$$

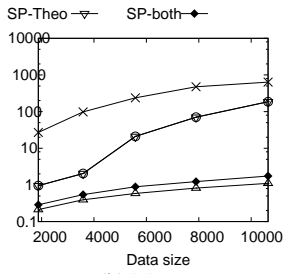

(b) Memory
Effect of $\epsilon_{t}$. The values used for $\epsilon_{t}$ are 0.2, 0.4, 0.6, 0.8 and 1 in radians $(|T|$ is fixed to be $5 k)$. Figure 13 shows the results on Geolife. According to these results, $\epsilon_{t}$ affects the SP algorithms only. Specifically, the running times of all SP algorithms increase slightly when $\epsilon_{t}$ becomes larger. This is because a larger $\epsilon_{t}$ usually results in $G_{\epsilon_{t}}$ with more edges and thus the BFS process on $G_{\epsilon_{t}}$ needs more time.
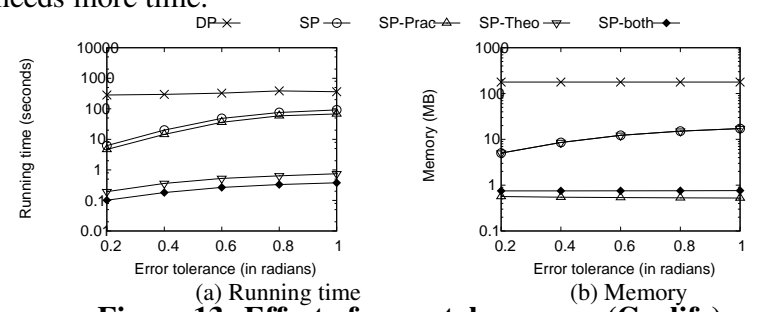

Figure 13: Effect of error tolerance $\epsilon_{t}$ (Geolife)

Compression Rate. We also study the effect of $\epsilon_{t}$ on the size ratio which is defined to be equal to $\sum_{T^{\prime} \in \mathcal{D}^{\prime}}\left|T^{\prime}\right| / \sum_{T \in \mathcal{D}}|T|$, where
$\mathcal{D}$ is the set of raw trajectories and $\mathcal{D}^{\prime}$ is the set of the corresponding simplified trajectories. Note that a smaller size ratio means a higher compression rate. The results are shown in Figure 11. We have the following observations. First, the size ratio decreases significantly when we increase the tolerance from 0 slightly. This is good since it implies that under DPTS, the trajectory data could be simplified significantly with a small error. Second, we observe that the size ratio is strictly smaller than 1 (e.g., it is about 0.9 for the Geolife datasets) even if the error tolerance is set to be 0 . This implies that the real-life trajectories usually involve a certain degree of redundancy and could be simplified without incurring any error.

Scalability Test. Figure 14 shows the results of the scalability test on the optimal algorithms. We only show the results of SP-theo and SP-both since the other optimal algorithms are not scalable on large datasets due to their expensive time complexities. According to these results, both SP-theo and SP-both are scalable to large trajectory datasets with millions of positions, and SP-both runs slightly faster than SP-theo. It is noted that SP-both occupies significantly less memory than SP-theo and thus SP-both is more scalable than SP-theo. This is because SP-both does not materialize $G_{\epsilon_{t}}$ explicitly while SP-theo does.

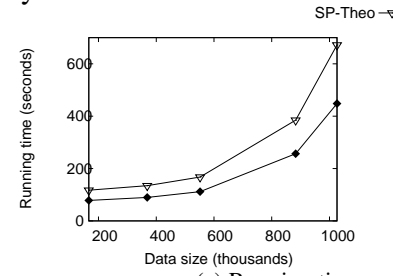

(b) Mem Running time
Figure 14: Scalability test (Geolife)

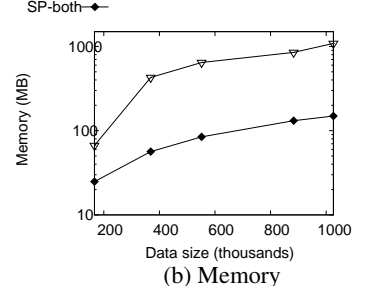

\subsection{Performance Study of the Approx. Algs}

In this part, we study the effects of $|T|$ and $\epsilon_{t}$ on the approximate algorithms. We use 3 measures, namely the running time, the memory and the approximation error. The approximation error of an approximate algorithm is defined to be $\left|T^{\prime}\right| /\left|T^{*}\right|$, where $T^{\prime}$ is the simplified trajectory returned by the approximate algorithm on a given raw trajectory and $T^{*}$ is the simplified trajectory returned by an optimal algorithm on the same raw trajectory.

Effect of $|T|$. The values used for $|T|$ are around $200 k, 400 k$, $600 k, 800 k$ and $1000 k$ ( $\epsilon_{t}$ is fixed to be 1$)$. Figure 15 shows the results. According to these results, Intersect is the fastest, which is at least 1 order of magnitude faster than other approximate algorithms. This is because Intersect runs in linear time while the other algorithms run in quadratic time in the worst case [16]. Besides, Intersect occupies the least memory and Greedy occupies slightly more memory than Intersect (though the difference in Figure 15(b) is not obvious).

Effects of $\epsilon_{t}$. Figure 16 shows the effects of $\epsilon_{t}$ on the approximate algorithms, where we vary $\epsilon_{t}$ with $0.2,0.4,0.6,0.8$ and 1 $(|T|$ is fixed to be $500 k$ ). According to these results, Greedy runs faster than Split and Merge with small $\epsilon_{t}$ 's. This is because with a smaller $\epsilon_{t}$, it is less likely that a long sequence of consecutive segments could be approximated with one segment and thus the cost 


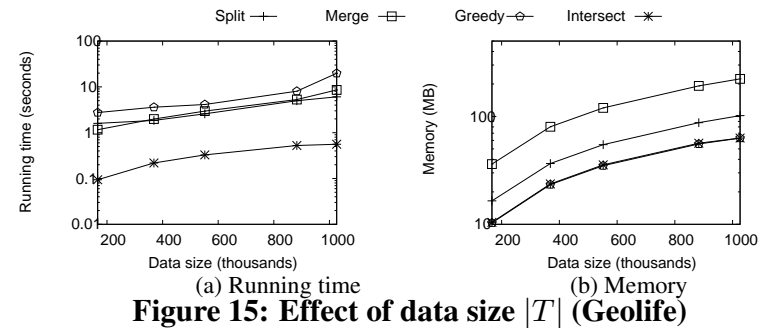

of checking the error of the segment linking the start position and the end position is small.

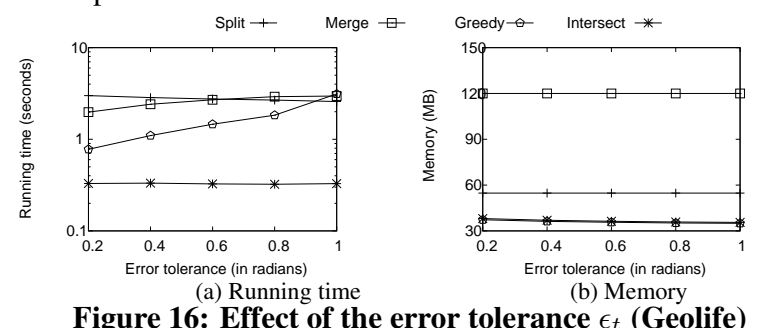

Compression Rate \& Approximation Error. Figure 17(a) shows the results on the compression rates of the approximate algorithms. We also show the theoretical bound of the size the trajectory returned by Intersect (Lemma 9). Figure 17(b) shows the results on the approximate errors of the approximate algorithms. Both these results verify our Intersect algorithm.
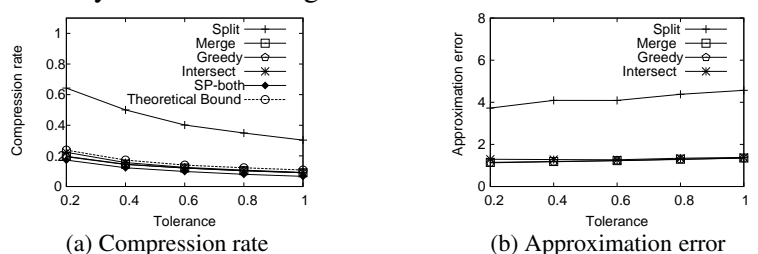

(b) Approximation error

Figure 17: Compression rates and approximate errors of the approximate algoritms (Geolife)

Scalability Test. The largest trajectory in our real datasets contains around $2 \mathrm{M}$ positions only. In order to generate larger trajectories, we concatenate multiple trajectories in ascending order of their time stamps into one. Figure 18 shows the results of the scalability test on the approximate algorithms. According to these results, Intersect is very fast on large datasets with more than $20 \mathrm{M}$ positions. For example, Intersect runs in $13.3 \mathrm{~s}$ on the trajectory with $24,876,978$ positions. In contrast, the running times of other approximate algorithms increase much faster when $|T|$ increases.

\section{CONCLUSION}

In this paper, we propose direction-preserving trajectory simplification, which has not been studied in the literature, as a novel alternative to the traditional position-preserving trajectory simplification. We propose an optimal algorithm called $S P$ and an approximate algorithm called Intersect. We conducted experiments to show the efficiency and the scalability of our proposed methods. There are many possible future directions. Firstly, it is interesting to study how to extend our algorithms when each segment is associated with a weight. Secondly, it is good to study how to simplify trajectories when positions are associated with labels (e.g., restaurants and gasoline stations).

Acknowledgements: We are grateful to the anonymous reviewers for their constructive comments on this paper. The research of Cheng Long and Raymond Chi-Wing Wong is supported by grants DAG12EG07-7 and FSGRF13EG27, and the research of H. V. Jagadish is supported in part by NSF grant IIS-1250880.

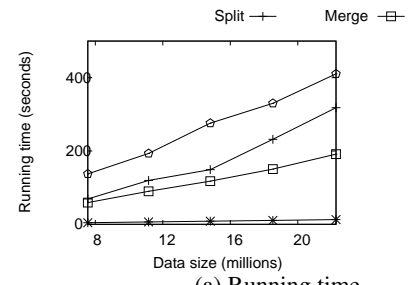

(a) Running time

Figure 18: Scalability test (Geolife)

\section{REFERENCES}

[1] S. Brakatsoulas, D. Pfoser, R. Salas, and C. Wenk. On map-matching vehicle tracking data. In $V L D B^{\prime} 05$, pages 853-864.

[2] S. Brakatsoulas, D. Pfoser, and N. Tryfona. Modeling, storing and mining moving object databases. In IDEAS'04, pages 68-77.

[3] H. Cao, O. Wolfson, and G. Trajcevski. Spatio-temporal data reduction with deterministic error bounds. The VLDB Journal, 15(3):211-228, 2006.

[4] M. Chen, M. Xu, and P. Fränti. A fast $o(\mathrm{n})$ multiresolution polygonal approximation algorithm for gps trajectory simplification. IEEE Transactions on Image Processing, 21(5), 2012.

[5] Y. Chen, K. Jiang, Y. Zheng, C. Li, and N. Yu. Trajectory simplification method for location-based social networking services. In Proceedings of the 2009 International Workshop on Location Based Social Networks, pages 33-40. ACM, 2009.

[6] D. Douglas and T. Peucker. Algorithms for the reduction of the number of points required to represent a digitized line or its caricature. The Canadian Cartographer, 11(2):112-122, 1973.

[7] F. Giannotti, M. Nanni, F. Pinelli, and D. Pedreschi. Trajectory pattern mining. In SIGMOD'07, pages 330-339.

[8] J. Gudmundsson, J. Katajainen, D. Merrick, C. Ong, and T. Wolle. Compressing spatio-temporal trajectories. Algorithms and Computation, pages 763-775, 2007.

[9] C.-C. Hung, W.-C. Peng, and W.-C. Lee. Clustering and aggregating clues of trajectories for mining trajectory patterns and routes. The VLDB Journal, pages 1-24, 2011.

[10] G. Kellaris, N. Pelekis, and Y. Theodoridis. Map-matched trajectory compression. Journal of Systems and Software, 2013.

[11] A. Kolesnikov. Efficient online algorithms for the polygonal approximation of trajectory data. In $M D M^{\prime} 11$, pages 49-57.

[12] R. Lange, T. Farrell, F. Durr, and K. Rothermel. Remote real-time trajectory simplification. In PerComm'09, pages 1-10, 2009.

[13] J. G. Lee, J. Han, and X. Li. Trajectory outlier detection: A partition-and-detect framework. In ICDE'08, pages 140-149.

[14] J. G. Lee, J. Han, X. Li, and H. Gonzalez. Traclass: trajectory classification using hierarchical region-based and trajectory-based clustering. PVLDB'08, 1(1):1081-1094, 2008.

[15] J. G. Lee, J. Han, and K. Y. Whang. Trajectory clustering: a partition-and-group framework. In SIGMOD'07, pages 593-604.

[16] C. Long, R. C.-W. Wong, and H. Jagadish. Direction-preserving trajectory simplification (technical report). In http://www.cse.ust.hk/ raywong/paper/trajectory.pdf, 2013.

[17] N. Meratnia and R. de By. Spatiotemporal compression techniques for moving point objects. EDBT'04, pages 561-562.

[18] J. Muckell, J. H. Hwang, V. Patil, C. T. Lawson, F. Ping, and S. Ravi. Squish: an online approach for gps trajectory compression. In COM.Geo'11, pages 13:1-13:8.

[19] D. Patel, C. Sheng, W. Hsu, and M. L. Lee. Incorporating duration information for trajectory classification. In $I C D E^{\prime} 12$, pages 1132-1143.

[20] N. Pelekis, I. Kopanakis, G. Marketos, I. Ntoutsi, G. Andrienko, and Y. Theodoridis. Similarity search in trajectory databases. In TIME'07, pages 129-140.

[21] M. Potamias, K. Patroumpas, and T. Sellis. Sampling trajectory streams with spatiotemporal criteria. In SSDBM’06, pages 275-284.

[22] M. Singh, Q. Zhu, and H. Jagadish. Swst: A disk based index for sliding window spatio-temporal data. In ICDE'12, pages 342-353.

[23] J. Yuan, Y. Zheng, X. Xie, and G. Sun. Driving with knowledge from the physical world. In KDD'11, pages 316-324.

[24] Y. Zheng, X. Xie, and W. Y. Ma. Geolife: A collaborative social networking service among user, location and trajectory. IEEE Data Engineering Bulletin, 33(2):32-40, 2010. 